\title{
Calculation of the Higgs Mass for Quark and Lepton Electric Charges Swap Lie-Groupoid
}

\author{
E Koorambas \\ Computational Applications, Group, Division of Applied Technologies, National Center for \\ Science and Research 'Demokritos', Aghia Paraskevi-Athens, Greece \\ E-mail: elias.koor@gmail.com
}

(March 7, 2022)

\begin{abstract}
Starting from the SU(2) group of weak interactions in the presence of Electric Charge Swap (ECS) symmetry, we show that ordinary and non-regular (ECS) leptons are related by the ECS rotational group $\mathrm{SO}(3)$. We find that many Standard Model (SM) algebras depend on the sin of the angle $\theta \mathrm{s}$ of the ECS rotational group SO (3). We call these ECSM algebras. Furthermore, the break of the gauge symmetry of the SM groupoid gives the massive ECS particle. We find that the ECS particle masses are related with the SM particle masses by $\sin \theta_{s}$. We also investigate the finite subgroups of the ECS Möbius transformations. We find that $\sin \theta_{\mathrm{S}}$ could be derived from the ECS dihedral group $\mathrm{D}_{\mathrm{F}}$, which refers to the symmetry of the fermionic polygon (F-gon). The average value of the anchor of the $\mathrm{SM}$ algebroid depends on the fermionic Catalan numbers $\left(\mathrm{C}_{\mathrm{F}}\right)$. Finally, we find that the ECS physics at loop level differs the SM physics. The ECSM mass is suppressed by the $\mathrm{C}_{\mathrm{F}}$ numbers. For 24 fermions, the calculated one-loop radiative correction to the bare Higgs mass $\mu$ is $125,6 \mathrm{GeV}-\mathrm{a}$ value very close to the experimental one.
\end{abstract}

PACS numbers: 11.30.-j, 02.20.-a, 11.25.Uv

Key-words: Higgs mass, Group Theory, Hypothetical Particles

\section{Introduction}

In the electroweak $(\mathrm{EW})$ theory, $\mathrm{SU}(2)_{\mathrm{L}} \times \mathrm{U}(1)_{\mathrm{Y}}[1]$ is broken down to the electromagnetic gauge group $\mathrm{U}(1)_{\mathrm{em}}$ by the vacuum expectation value of an elementary scalar field $\varphi$. This scalar field should be part of the Grand Unification Theory (GUT)[2]. To produce a vacuum expectation value of a size that imparts to the observed $\mathrm{W}$ and $\mathrm{Z}$ boson a mass of 82 and 91 $\mathrm{GeV}$, respectively, the Standard Model (SM) Higgs scalar field must obtain a negative mass term, of a size [1]:

$-\mu_{0}^{2}=-(100)^{2}$. 
Equation (1) is subjected to additive renormalization. Beyond tree level, the radiative corrections to the Higgs mass for the Higgs $(H)$, top quark $\left(t-t^{-}\right)$, and boson $(W, Z)$ loops in the SM [4-6], are shown in Figure 1:

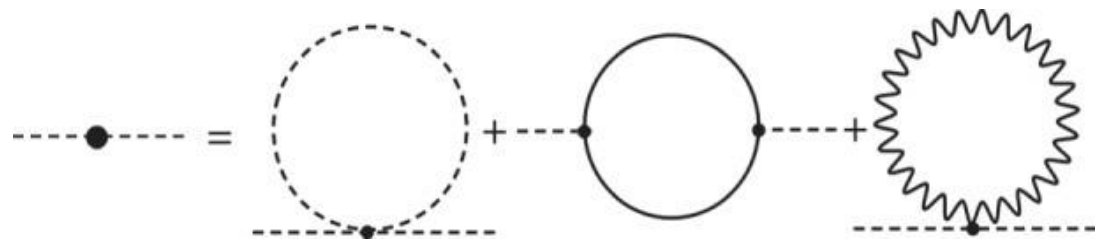

Fig. 1. One-loop corrections to the Higgs mass. The diagrams for the Higgs $(H)$, top quark ( $t$ $\left.t^{-}\right)$, and boson $(W, Z)$ loops are quadratically divergent, and make the Higgs mass highly UVsensitive [7] C. Grojean, Philos Trans A Math Phys Eng Sci. 2015 Jan 13; 373(2032): 20140042).

Each diagram in Figure 1 shows a contribution to the self-energy $i \Sigma\left(p^{2}\right)$, where $p$ is the fourmomentum of the external particle [8]:

$$
M_{H}^{2}=\mu_{0}^{2}+\sum\left(M_{H}^{2}\right) .
$$

In Equation (2), $\mu_{0}$ is the bare Higgs mass. Using a cutoff to regularize the bare amplitudes [8], these radiative corrections lead to the well-known mass correction:

$M_{H}^{2}=\mu_{0}^{2}+\frac{3 \Lambda_{C}^{2}}{32 \pi^{2} v^{2}}\left[M_{H}^{2}+2 M_{W}^{2}+M_{Z}^{2}-4 m_{t}^{2}\right]+\ldots$

where $m_{t}, M_{W, Z}$, and $M_{H}$ are the mass of the top quark, $W, Z$ bosons, and Higgs bosons $(H)$, respectively, $v$ is the vacuum expectation value of the Higgs potential in the SM, and $\Lambda_{C}$ is the cutoff energy scale $\Lambda_{C}$ [4-6]. The dots indicate logarithmic corrections at the cutoff energy scale $\Lambda_{C}$, as well as contributions independent of the cutoff energy scale, in the large $\Lambda_{C}$ limit [8].

$\Lambda c$ can be the energy scale of a new physics coupled to the SM one. In particular, $\Lambda c$ can even be the Planck mass scale, $M_{p}$. On the other hand, it is unnatural to have $\Lambda_{c} \gg \mathrm{m}_{\mathrm{w}}$, since everything is defined by the EW scale [9]. Equation (3) is the source of the usual fine-tuning problem in the SM: if $\Lambda_{c} \sim m_{W}$, then we must suppose that the tree-level $\mu^{2}$ and the loop contributions cancel each other in order for $\left|\mu_{0}{ }^{2}\right|$ to be $\sim m^{2}{ }_{W}$ [9]. After the discovery of the Higgs boson (of a mass of $125 \mathrm{GeV}$ ) in the ATLAS and CMS experiments [10, 11], the SM would be a perfect theory but from the fine-tuning problem. There are two general ways to solve this problem: either by evoking some (super)symmetry to cancel out the huge terms [12], or by introducing some new physics, such as large extra dimensions [13], composite Higgs models[14], etc., at a scale not very far from the electroweak one, thus making the $\Lambda_{c}$ to be small [7]. The observation of a light Higgs boson with properties consistent with the SM $[10,11]$ has motivated much reexamination of the notion of the naturalness problem [7].

It is well known that the supersymmetric extension of the SM can solve the fine-tuning problem. Despite many efforts to search for supersymmetry at the Large Hadron Collider (LHC) experiments, the current LHC data do not offer unequivocal proof for the production of supersymmetric particles [15]. For this reason, we investigate the possibility of solving the fine-tuning problem without supersymmetry. 
In the SM, mass is generated due to spontaneous symmetry breaking in the Higgs sector. While the Higgs boson mass was introduced by Peter Higgs et al. [16] from the outset, the tachyon mass term breaks the scale invariance (conformal symmetry) explicitly [16]. However, if the conformal symmetry could be broken sufficiently 'softly' so that symmetry is restored at high energies, the Higgs mass would still be protected from the largest radiative corrections necessitated by the highest energies $[17,18,19]$.

Recently P. Grang' et.al [8] re-analysed the perturbative radiative corrections to the Higgs mass within the SM in the light of the Taylor-Lagrange renormalisation scheme. This scheme naturally leads to completely finite corrections, depending on an arbitrary scale. Nima Arkani-Hamed et.al [20] proposed the N-naturalness model, which presents a new solution to the electroweak hierarchy problem. The authors introduced $\mathrm{N}$ copies of the SM, with varying values of the Higgs mass parameter. This proposition generally yields a sector whose weak scale is parametrically removed from the cutoff by a factor of $1 / \sqrt{ } \mathrm{N}$.

In the framework of fine symmetries, the Monster Group is related to the symmetries of a particular bosonic string theory on the Leech lattice [21]. However, there is no physical reason for the presence of the Monster Group or its subgroups in the Lagrangian: its appearance may merely be a coincidence. Another coincidence is that, in reduced Planck units, the Higgs mass is approximately $48|M|^{-1 / 3}=125.5 \mathrm{GeV}$ (where $|M|$ is the order of the Baby Monster group). This suggests that the small size of the Higgs mass may be due to a redundancy caused by a symmetry of the extra dimensions, which must be divided out [22]. It is also possible that the hierarchy problem is a specific manifestation of Bayesian statistics[23-27] .

In the Fine-Tuning Naturalness (FTN) problem, we suppose that bare SM parameters are physically interpreted as "fundamental parameters," analogous to the microscopic lattice parameters of a Condensed Matter (CM) system [28]. Alternatively, bare SM parameters can be interpreted as unphysical "auxiliary parameters": if the Wilsonian Renormalization Group (RG) transformations are interpreted as invertible re-parametrisations, the bare parameters in High Energy Physics (HEP) and Condensed Matter Physics (CMP) are formally - but not physically -analogous [28]. Furthermore, it is possible that neither the bare mass, $\mu_{0}$, nor the radiative corrections, $\delta M^{2}$, are directly measurable. Since these are probably not physical, there is no coincidence to be explained (Wetterich 1984[29]), (Bianchi \& Rovelli 2010[30]).

For quarks and leptons, an Electric Charge Swap (ECS) symmetry has been proposed by the author [31]. A family of particular transformations may be continuous (e.g., the rotation of a circle) or discrete (e.g., the reflexion of a bilaterally symmetrical figure, or the rotation of a regular polygon) [31-34], [35]. ECS transformation between ordinary families of leptons produces heavy, neutral, non-regular leptons of an O-order mass (TeV). These particles may form cold dark matter [31]. Furthermore, the ECS symmetry could explain certain properties of lepton families within the framework of superstring theories [36-39]. Recently, AWollmann Kleinert and F. Bulnes, based on ECS symmetry (in this case, leptons [31]), proposed that leptons are the subtle Fermions [35].

From the mathematical point of view, in R-Category - a category theory with invertible morphisms [40] - the geometric structures under consideration are always associated with local Lie brackets [, ] on sections of some vector bundles (Lie algebroids [41,42]). Based on [41-43], in this article, we study the structure of transitive Lie algebroids as a mathematical framework for generalizing the formulation of a gauge theory through an action functional: 
the integral of a differential form on the algebroid [44]. On Atiyah Lie algebroids [45], the space of ordinary connections 1-forms corresponds with the Ehresmann connections on a principal fiber bundle P (see the S. Lazzarini and T. Masson in [46,47]). Cédric Fournel (2013) [44] proved that transitive Lie algebroids equipped with generalized connections contain scalar fields as algebraic parameters. These parameters, absent in differential geometry, have a role similar to that of the scalar field in the Higgs mechanism [16]. In higher-dimensional spacetime, the Double Field Theory (DFT) is a gravity theory with manifest T duality (Hull-Zwiebach, 2009 [48]). The DFT has gauge symmetry (described by the $C$ bracket ([ , ] ${ }_{C}$ : see[48])), which defines the Vaisman-algebroid (Vaisman, 2013[49]).

This paper is structured as follows: In section 2, taking the SU(2) group of weak interactions in the presence of ECS symmetry as a starting point, we show that ordinary and non-regular (ECS) leptons are related by the ECS rotational SO(3) group. In section 3, we investigate a version of the SM algebroid whose anchor map depends on the sin of the ECS angle $\theta_{\mathrm{s}}$ of the ECS rotational SO (3) group. We find many SM algebras that depend on $\sin \theta_{\mathrm{s}}$; we call these algebras ECSM algebras. Furthermore, the broken gauge symmetry of the SM groupoid gives the massive ECS particle. We find that the ECS particle mass is related with the SM particle mass by $\sin \theta_{\mathrm{s}}$. In section 4 , we investigate the finite subgroups of the ECS-Möbius transformations. In this case, $\sin \theta_{\mathrm{s}}$ could be originated from the ECS dihedral group $\mathrm{D}_{\mathrm{F}}$, which refers to the symmetry of the Fermionic polygon (F-gon). The average value of the anchor of the SM algebroid then depends on the fermionic Catalan numbers, $\mathrm{C}_{\mathrm{F}}$. Finally, in section 5, we find that the ECS physics at loop-level differs from the SM physics: the ECSM mass is suppressed by the numbers $\mathrm{C}_{\mathrm{F}}$. For 24-fermions, the calculation of one-loop radiative corrections to the bare Higgs mass $\mu$ is $125,6 \mathrm{GeV}$, which is very close to the experimental value.

\section{The global ECS symmetry for quarks and leptons}

Hypothetical non-regular leptons are, a) a zero-charged version of the electron, $\tilde{e}^{0}$, and, b) a positively charged version of the electron neutrino, $\tilde{v}_{e}^{+}$. Non-regular leptons can, therefore, be obtained from the swap of electric charge between electrons and electron neutrinos in the internal space. We call these proposed non-regular leptons, electric charge swap (ECS) leptons [19].

Although ECS leptons have the same mass as ordinary-family leptons, they are distinguished from the latter by their different lepton numbers ( $L_{s}=1$ for ordinary leptons; $\bar{L}_{s}=-1$ for ordinary antileptons) and their electric charge (positive or neutral for ordinary leptons; negative or neutral for ordinary antileptons). We hypothesize that ECS leptons are produced from ordinary-family leptons when the latter enter the internal space: in these conditions, the properties of ordinary-family leptons change profoundly as these leptons lose their 'individuality' and swap their electric charge [31-34]. To formulate the swap of electric charge between ordinary leptons, we have to look for a global symmetry that characterises the swap process in the framework of 2-internal dimensions [31-34]. We consider the 2-sphere $S^{2}$ as a quotient space $\left(S^{2} \equiv S U(2)_{L} / U(1)_{\Upsilon}\right)$, and express it in terms of the new symmetry between the original lepton and the new ECS lepton doublets. We do this in the following steps [31-34]: First, we observe that both the ordinary lepton doublet, $l_{0}\left(x^{v}\right)=\left(\mathrm{e}_{L}^{-}, v_{e}\right)$, and 
the ECS lepton doublet, $\tilde{l}_{0}\left(x^{\nu^{\prime}}\right)=\left(\tilde{\mathrm{e}}_{L}^{0}, \tilde{v}_{e}^{+}\right)$, can form the fundamental representation of $S U(2)_{L}[50]$. This fundamental representation is given by:

$\left[I_{\alpha}, I_{\beta}\right]=i \varepsilon_{\alpha \beta \gamma} I_{\gamma}$

The generators are denoted as:

$$
I_{\alpha}=\frac{1}{2} \tau_{\alpha}
$$

where

$$
\tau_{1}=\left(\begin{array}{ll}
0 & 1 \\
1 & 0
\end{array}\right), \tau_{2}=\left(\begin{array}{cc}
0 & -i \\
i & 0
\end{array}\right), \tau_{3}=\left(\begin{array}{cc}
1 & 0 \\
0 & -1
\end{array}\right)
$$

are the isospin versions of Pauli matrices.

The action of the latter on the new lepton states is represented by:

$$
\tilde{e}_{L}^{0}=\left(\begin{array}{l}
1 \\
0
\end{array}\right), \tilde{v}_{e}^{+}=\left(\begin{array}{l}
0 \\
1
\end{array}\right) .
$$

To link the two distinct types of lepton, ordinary and ECS leptons, we assume that neither the ordinary $(L)$ nor the ECS $\left(L_{s}\right)$ lepton numbers are conserved, while the overall number of leptons is conserved obligatorily.

$$
\begin{aligned}
& L_{\text {overall }}=L_{s}+L=0 . \\
& L_{s}=\bar{L}, L_{s}\left(v_{e}^{+}\right)=\bar{L}\left(\mathrm{e}^{+}\right)=-1 . \\
& \bar{L}_{s}=L, \bar{L}_{s}\left(\tilde{\mathrm{e}}^{0}\right)=L\left(v_{e}\right)=1 .
\end{aligned}
$$

The quantum numbers of the new ECS leptons are given in Table 1 [31-34].

Table 1. Quantum numbers (weak ECS isospin $I_{s}$, charge Q, ECS hypercharge $Y_{s}$, ECS lepton number $\mathrm{L}_{\mathrm{S}}$ ) of the ECS leptons $\tilde{e}_{L}^{0}, \tilde{v}_{e}^{+}$.

\begin{tabular}{lccccc}
\hline New lepton & $\mathrm{I}$ & $\mathrm{Is}-\mathrm{Z}$ & $\mathrm{Q}$ & $\mathrm{Y}_{\mathrm{s}}$ & $\mathrm{L}_{\mathrm{s}}$ \\
\hline$\tilde{v}_{e}^{+}$ & $1 / 2$ & $1 / 2$ & 1 & 1 & -1 \\
& & & & & \\
\hline$\tilde{e}_{L}^{0}$ & $1 / 2$ & $-1 / 2$ & 0 & 1 & -1 \\
\hline
\end{tabular}

The next step is to define the group transformation that can account for the swap of electric charges between the electron and electron neutrino particles. The global ECS transformation must be derived from a transformation from: 
1) $S U(2)_{I} / U(1)_{Y}$, in which the fundamental representation of $S U(2)_{I}$ is $l_{0}\left(x^{v}\right)=\left(\mathrm{e}_{L}^{-}, v_{e}\right)$, and $U(1)_{Y}$ is the symmetric group generated by hypercharge $Y=-1$

to

2) $S U(2)_{I_{s}} / U(1)_{Y_{s}}$, in which the fundamental representation of $S U(2)_{I_{s}}$ is $\tilde{l}_{0}\left(x^{\nu^{\prime}}\right)=\left(\tilde{\mathrm{e}}_{L}^{0}, \tilde{v}_{e}^{+}\right)$, and $U(1)_{Y_{S}}$ is the symmetric group generated by swap hypercharge $Y_{S}=1$.

The quotient space $S U(2) / U(1)$ is diffeomorphic to the unit 2-sphere $S^{2}$. Consequently, the swap of electric charges between electrons and electron neutrinos must be an automorphism of the 2-sphere to itself [31-34]. Since the two internal dimensions are endowed with the Fubini-Study ${ }^{[1]}$ metric [51], [52], not all Möbius transformations (e.g., dilations and translations) are isometries. Therefore, the automorphism from $S^{2} \equiv S U(2) / U(1)$ to itself, which causes the electric charge swap between the electrons and electron neutrinos, is given by the isometries that form a proper subgroup of the group of projective linear transformations $P G L_{2}(\hat{C})_{(C h a r g e)}$ —namely $P S U_{2(C h a r g e)}$. Subgroup $P S U_{2(\text { Charge })}$ is isomorphic to the global rotation group $S O(3)_{E C S}$ [51], [52],[ 31-34], which is the isometric group of the unit sphere in three-dimensional real space $R^{3}$. The automophism of the Riemann sphere $\hat{C}$ is given by:

$$
\begin{aligned}
& \operatorname{Rot}_{(E C S)}(\hat{\mathbb{C}})=P S U_{2(C h a r g e)}=S O(3)_{E C S}, \\
& \hat{\mathbb{C}}=\mathbb{C} \cup \infty=S^{2},
\end{aligned}
$$

where $\hat{\mathbb{C}}$ is the extended complex plane, $P S U_{2(C h \arg e)}$ is the proper subgroup of the projective linear transformations, and global swap symmetry, $S O(3)_{E C S}$, is the group of rotations in three-dimensional vector space $R^{3}$. This can be consigned in the double fibration on a vector bundle of lines $\mathfrak{I}^{2}$ in the extended space (ad infinitum), that is to say, $\hat{\mathbb{C}}=\mathbb{C} \cup \infty$. The universal cover of $S O(3)_{E C S}$ is the special unitary group $S U(2)_{I\left(I_{S}\right)}$ [31-34]. This group is also differomorphic to the unit 3 sphere $S^{3}$. We regard the ordinary and ECS leptons as different electric charge states of the same particle - analogous, that is, to the proton-neutron isotopic pair. Finally, in terms of global rotational symmetry between the original lepton and the proposed ECS lepton, the ECS 2 sphere $S_{E C S}^{2}$ is given by:

$$
S_{E C S}^{2} \equiv S U(2)_{I\left(I_{S}\right)} / U(1)_{Y\left(Y_{S}\right)}[19],
$$

\footnotetext{
${ }^{[1]}$ The round metric of the 2-sphere can be expressed in stereographic coordinates as:

$G=\frac{d y_{1}^{2}+d y_{2}^{2}}{\left(1+\varepsilon^{2}\right)^{2}}$, where $\varepsilon=\sqrt{y_{1}^{2}+y_{2}^{2}}$. The metric $G$ is the Fubini-Study metric of the 2-sphere [51], [52]
} 
where $S U(2)_{I\left(I_{S}\right)}$ is the special unitary group and $U(1)_{Y\left(Y_{s}\right)}$ is the symmetric group generated by hypercharge $Y\left(Y_{s}\right)$. Similar with hypothetical non-regular leptons, hypothetical nonregular quarks are, a) an 1/3-electrically charged version of the up ( $\alpha$ ) quark types, $\alpha^{\sim}$, and, b) a $-2 / 3$-electrically charged version of the down $(\kappa)$ quark types, $\kappa^{\sim}$. Non-regular quarks can, therefore, be obtained from the swap of electric charge between up and down quark types. We call these proposed non-regular quarks electric charge swap (ECS) quarks [31-34]. We regard ordinary and ECS quarks as different electric charge states of the same particle-analogous, that is, to the proton-neutron isotopic pair [31-34]. Some quantum numbers of the new ECS quarks are shown in Table 2.

Table 2: Quantum numbers of the proposed ECS quarks.

\begin{tabular}{|l|l|l|l|}
\hline $\begin{array}{l}\text { New } \\
\text { quarks }\end{array}$ & Q: electric charge & $\mathrm{I}_{\mathrm{sz}}:$ ECS isospin component & $\mathrm{B}_{\mathrm{s}}$ : Baryonic number \\
\hline $\mathrm{u}^{\sim}, \mathrm{c}^{\sim}, \mathrm{t}^{\sim}$ & $1 / 3$ & $-1 / 2$ & $-1 / 3$ \\
\hline $\mathrm{d}^{\sim}, \mathrm{s}^{\sim}, \mathrm{b}^{\sim}$ & $-2 / 3$ & $1 / 2$ & $-1 / 3$ \\
\hline
\end{tabular}

The simplest way to realise the global $\mathrm{SO}(3)_{\mathrm{ECS}}$ group provided by Equation (11) is by adding the ECS electron $\tilde{e}_{L}^{0}$ and grouping it together with the ECS electron neutrino $\tilde{v}_{e}^{+}$, electron antineutrino $\bar{v}_{e}$, and electron $e^{-}$into a triplet, as follows:

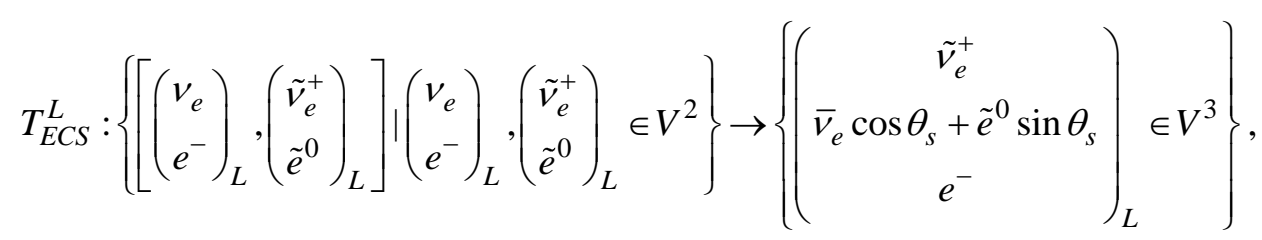

where $\theta_{s}$ is an arbitrary real parameter, independent of space-time coordinates, which represents the lepton and ECS quark mixing angle. There remains a left-hand singlet:

$$
\begin{aligned}
& S_{E C S}^{L}:\left\{\left[\left(e^{-}\right)_{R},\left(\tilde{v}_{e}^{+}\right)_{R}\right] \mid l\left(e^{-}\right)_{R},\left(\tilde{v}_{e}^{+}\right) \in V_{\text {row }}^{1}\right\} . \\
& \rightarrow\left\{\left(\bar{v}_{e} \cos \theta_{s}-\tilde{e}^{0} \sin \theta_{s}\right)_{L} \in \tilde{V}_{\text {row }}^{1}\right\}
\end{aligned}
$$

The muon $\left(\mu^{-}\right)$and its antineutrino $\left(\bar{v}_{\mu}\right)$, as well the tau $\left(\tau^{-}\right)$and its antineutrino $\left(\bar{v}_{\tau}\right)$, can be introduced in a manner similar to the above, at the cost of the four ECS leptons $\left(\tilde{\mu}_{L}^{0} \tilde{v}_{\mu}^{+}, \tilde{\tau}_{L}^{0} \tilde{v}_{\tau}^{+}\right)$. For this introduction, we have to add an 1/3-electrically charged version of the up $(\alpha)$ quark types (ECS- $\alpha$ quarks) and group them together with the $-2 / 3$-electrically

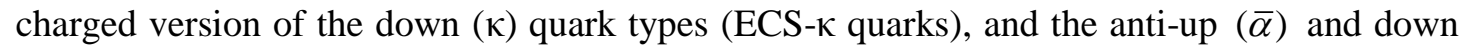

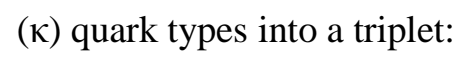

$$
T_{Q_{L}}=\left(\begin{array}{c}
\tilde{\alpha} \\
\bar{\alpha} \sin \theta_{s}+\tilde{\kappa} \cos \theta_{s} \\
\kappa
\end{array}\right)_{L} .
$$


There remains a left-hand singlet:

$$
S_{Q_{L}}=\left(\tilde{\kappa} \sin \theta_{s}+\bar{\alpha} \cos \theta_{s}\right)_{L}
$$

Equations (13) and (15) form the fundamental representation space of the $\mathrm{SO}(3)_{\mathrm{ECS}}$ group:

$$
\begin{aligned}
& \left\{\left(\begin{array}{c}
\tilde{v}_{e}^{+} \\
\bar{v}_{e} \sin \theta_{s}+\tilde{e}^{0} \cos \theta_{s} \\
e^{-}
\end{array}\right),\left(\begin{array}{c}
\tilde{v}_{\mu}^{+} \\
\bar{v}_{\mu} \sin \theta_{s}+\tilde{\mu}^{0} \cos \theta_{s} \\
\mu^{-}
\end{array}\right),\left(\begin{array}{c}
\tilde{v}_{\tau}^{+} \\
\bar{v}_{\tau} \sin \theta_{s}+\tilde{\tau}^{0} \cos \theta_{s} \\
\tau^{-}
\end{array}\right)\right\}_{L}, \\
& \left\{\left(\begin{array}{c}
\tilde{u} \\
\overline{\mathrm{u}} \sin \theta_{s}+\tilde{d} \cos \theta_{s} \\
d
\end{array}\right),\left(\begin{array}{c}
\tilde{\mathrm{c}} \sin \theta_{s}+\tilde{s} \cos \theta_{s} \\
s
\end{array}\right),\left(\begin{array}{c}
\tilde{t} \sin \theta_{s}+\tilde{b} \cos \theta_{s} \\
b
\end{array}\right)\right\}_{L} .
\end{aligned}
$$

Equations (17) and (18) are 3-component vectors for the linear combination between fermions and ECS fermions. The representative matrix of a general element of the $\mathrm{SO}(3)_{\mathrm{ECS}}$ group can be written as:

$$
O_{E C S}=\mathrm{e}^{-i \sum_{\alpha} \theta_{s}^{\alpha} X_{\alpha}}, \alpha=(1,2,3)
$$

with $\theta^{\alpha}=\left(\theta^{1}, \theta^{2}, \theta^{3}\right)$ being arbitrary real group parameters independent of space-time coordinates, and $O_{E C S}$ being an orthogonal $3 \times 3$ matrix:

$$
O_{E C S}^{T} O_{E C S}=I=O_{E C S} O_{E C S}^{T}
$$

The three basic ECS rotation matrices that rotate fermions by an angle $\theta_{s}{ }^{\alpha}(\alpha=1,2,3)$ about the $\mathrm{x}-, \mathrm{y}-$, or $\mathrm{z}$-axis in three internal dimensions can be explicitly written as follows:

$$
O_{E C S}=\mathrm{e}^{-i \theta_{s}^{\alpha} X_{\alpha}} \text {. }
$$

In this representative space, the representative matrices of the generators of the $\mathrm{SO}(3)_{\mathrm{ECS}}$ group are denoted by $X_{\alpha}(\alpha=1,2,3)$ :

$$
X_{1}=\frac{1}{\sqrt{2}}\left(\begin{array}{ccc}
0 & 1 & 0 \\
1 & 0 & 1 \\
0 & 1 & 0
\end{array}\right), \quad X_{2}=\frac{i}{\sqrt{2}}\left(\begin{array}{ccc}
0 & -1 & 0 \\
1 & 0 & -1 \\
0 & 1 & 0
\end{array}\right), \quad X_{3}=\left(\begin{array}{ccc}
1 & 0 & 0 \\
0 & 0 & 0 \\
0 & 0 & -1
\end{array}\right) \text {. }
$$

These matrices satisfy the following condition:

$$
\left[\mathrm{X}_{\alpha}, \mathrm{X}_{\beta}\right]=i C_{\alpha \beta \gamma} X_{\gamma}
$$

where $C_{\alpha \beta \gamma}$ are structure constants of the $\mathrm{SO}(3)_{\mathrm{ECS}}$ group. Generator $X_{\alpha}$ is Hermitian and traceless:

$$
X_{\alpha}^{\dagger}=X_{\alpha}, \operatorname{Tr}_{\alpha}=0
$$


To calculate the trace, we used the sum of the diagonal elements of the rotation matrix given by Equation (21):

$$
\operatorname{Tr}\left(O_{E C S}\right)=2 \cos \left\|\theta_{S}\right\|+1 \text {. }
$$

From Equation (25), it follows that the arbitrary absolute value of the ECS angle is:

$$
\begin{aligned}
& \theta_{s}=\left\|\theta_{s}\right\|=\arccos \left(\frac{\operatorname{Tr}\left(O_{E C S}\right)-1}{2}\right)=\frac{\pi}{2}-\arcsin \left(\frac{\operatorname{Tr}\left(O_{E C S}\right)-1}{2}\right), \\
& F\left(\theta_{S}\right) \equiv \sin \left\|\theta_{s}\right\|=\frac{\operatorname{Tr}\left(O_{E C S}\right)}{2}
\end{aligned}
$$

\section{The Standard Model of particle physics in the R-Category theory}

In general, the $\mathrm{U}(\mathrm{n})$ gauge group cannot be identified as a more familiar-looking manifold. It is an 'iterated extension' of the odd-dimensional sphere $S^{1}, S^{3}, \ldots, S^{2 n-1}$, and the rational homotopic equivalent to the product $S^{1} \times S^{3} \times \ldots \times S^{2 n-1}$ [53-55]. This means that the U(n) gauge group has the same rational co-homology and rational homotopy groups as this product; however, it is generally not homeomorphic or diffeomorphic to this product[53-55]. 'Iterated extension' means that the unitary groups fit into fiber sequences which are ultimately built from odd spheres, starting with:

$$
S U(\mathrm{n}) \rightarrow U(\mathrm{n}) \stackrel{\text { det }}{\rightarrow} S^{1},
$$

and continuing with:

$$
S U(\mathrm{n}-1) \rightarrow S U(\mathrm{n}) \rightarrow S^{2 n-1} .
$$

The first sequence is a short exact sequence of Lie groups and splits smoothly; therefore, the $\mathrm{U}(\mathrm{n})$ is diffeomorphic to $S U(\mathrm{n}) \times S^{1}$ [53-55]. In particular, the SM gauge group $\mathrm{U}(2)$ [5657] is diffeomorphic to the product $S^{3} \times S^{1}$ manifold. However, this is not an isomorphism of groups, since $U(n)$ is a semidirect rather than a direct product. The vector spaces of

$$
\sum_{\alpha} \varepsilon^{\alpha}(x) T_{\alpha}=\alpha(x) I+\beta(x)^{a} \tau_{a}, \alpha=(1,2,3),
$$

together with the commutator relation

$$
\begin{aligned}
& {\left[I_{2}, \tau_{\alpha}\right]=0,\left[\tau_{1}, \tau_{2}\right]=2 i \tau_{3}, \alpha=(1,2,3)} \\
& {\left[\tau_{2}, \tau_{3}\right]=2 i \tau_{1},\left[\tau_{3}, \tau_{1}\right]=2 i \tau_{2},}
\end{aligned}
$$

are referred to as the SM algebra $\aleph=u(2) \cong \mathbb{R}^{4} . I_{2}$ are the $2 \times 2$ unity matrix and the 3 tuple $\tau$ (Equation.(31)) of the Hermitian and traceless matrices, respectively; $\varepsilon^{a}(x)$ are the real arbitrary group parameters of the local $\mathrm{U}(2)$ gauge group; and $\alpha(\mathrm{x})$ and $\beta(\mathrm{x})$ are the real arbitrary group parameters of the local $\mathrm{SU}(2)$ and $\mathrm{U}(1)$ gauge groups, respectively.

From the mathematical point of view, in R-Category - a category theory with invertible morphisms [40] — the geometric structures we consider here are always associated with local 
Lie brackets [, ] on sections of some vector bundles (Lie algebroids). A Lie algebroid over a manifold B is defined to be a vector bundle A over B with a Lie algebra structure on its space of smooth sections [, ], together with a bundle map, $\rho$ (called the anchor of the Lie algebroid), from A to the tangent bundle TB. Here, we definite the ECS-Standard Model (ECSM) algebra $\tilde{\aleph}=\tilde{u}(2) \cong \mathbb{R}^{4}$ from the $\mathrm{SM}$ algebroid $\mathrm{A}_{\mathrm{SM}}$ over the product $M=S^{3} \times S^{1}$ manifold which satisfies the conditions:

$$
\begin{aligned}
& {\left[\rho\left(\mathrm{I}_{2}\right), \rho\left(\tau_{\alpha}\right)\right]=\rho\left(\left[\mathrm{I}_{2}, \tau_{\alpha}\right]\right)} \\
& {\left[\mathrm{I}_{2}, \varphi \tau_{\alpha}\right]=\varphi\left[\mathrm{I}_{2}, \tau_{\alpha}\right]+\left(\rho\left(\mathrm{I}_{2}\right) \cdot \varphi\right) \tau_{\alpha} \quad, \alpha=(1,2,3)} \\
& {\left[\rho\left(\tau_{\alpha}\right), \rho\left(\tau_{\beta}\right)\right]=\rho\left(\left[\tau_{\alpha}, \tau_{\beta}\right]\right)} \\
& {\left[\tau_{\alpha}, \varphi \tau_{\beta}\right]=\varphi\left[\tau_{\alpha}, \tau_{\beta}\right]+\left(\rho\left(\tau_{\alpha}\right) \cdot \varphi\right) \tau_{\beta}, \quad \beta=(1,2,3)}
\end{aligned}
$$

(with $\tau_{\alpha}$ being smooth sections of $\mathrm{A}_{\mathrm{SM}}$, and $\phi$ being a smooth function on $M=S^{3} \times S^{1}$ ), when the anchor's smooth multiplication factor of the SM-algebroid, $A_{S M}$, is given by Equation (26), and the ECS generators are derived from the SM generators by the anchor map:

$$
\begin{aligned}
& \tilde{I}_{2}=\rho\left(I_{2}\right)=\mathrm{F}\left(\theta_{s}\right) I_{2}=I_{2} \sin \theta_{s} \in \Gamma(T M), \\
& \tilde{\tau}_{2}=\rho\left(\tau_{2}\right)=\mathrm{F}\left(\theta_{s}\right) \tau_{2}=\tau_{2} \sin \theta_{s} \in \Gamma(T M) .
\end{aligned}
$$

In the above equations, $\Gamma(T M)$ indicates the sections of the tangent bundle TM,

$$
F=\mathrm{F}\left(\theta_{s}\right)=\sin \theta_{s}
$$

indicates the smooth function on $S^{3} \times S^{1}$, and $\theta_{s}$ are the arbitrary ECS-angles if we parametrise the unit 3 -sphere by hyperspherical coordinates $\left(x_{0}, x_{1}, x_{2}, x_{3}\right)$ and use $\left(\psi, \theta_{s}, \varphi\right)$. By restricting the domain of Equation (26), we obtain:

$$
\begin{aligned}
& \sin :\left[-\frac{\pi}{2}, \frac{\pi}{2}\right] \rightarrow[-1,1], \\
& \theta_{s} \mapsto \sin \theta_{s}
\end{aligned}
$$

This function is both one-one and onto; therefore, it has inverse function:

$$
\begin{aligned}
& \sin ^{-1}:[-1,1] \rightarrow\left[-\frac{\pi}{2}, \frac{\pi}{2}\right], \\
& \theta_{s} \mapsto \sin ^{-1} \theta_{s}
\end{aligned}
$$

By Equations (56) and (57), the anchor of the SM-algebroid, $\mathrm{A}_{\mathrm{SM}}$, is thus both an one-one and onto map between the SM and ECS generators.

$$
\left[\rho\left(T_{\alpha}\right), \rho\left(T_{\beta}\right)\right]=\left[\mathrm{F}\left(\theta_{s}\right) T_{\alpha}{ }^{\prime}, \mathrm{F}\left(\theta_{s}\right) T_{\beta}\right]=\mathrm{F}\left(\theta_{s}\right)^{2}\left[T_{\alpha}{ }^{\prime}, T_{\beta}\right]=\frac{\mathrm{F}\left(\theta_{s}\right)^{2}}{\mathrm{~F}\left(\theta_{s}\right) \pm \varepsilon}\left[T_{\alpha}, T_{\beta}\right]=i \frac{\mathrm{F}\left(\theta_{s}\right)^{2}}{\mathrm{~F}\left(\theta_{s}\right) \pm \varepsilon} T_{\gamma}
$$


$T_{\alpha}{ }^{\prime} \rightarrow \frac{T_{\alpha}}{\mathrm{F}\left(\theta_{s}\right) \pm \varepsilon}$; the changes of scale of the generator $T_{\alpha}$, and $\varepsilon$ an infinitesimal positive quantity. $\rho\left(\left[T_{\alpha}, T_{\beta}\right]\right)=\rho\left(i T_{\gamma}\right)=i F\left(\theta_{s}\right) T_{\gamma}(59)$.For $\varepsilon \rightarrow 0$ by Equations (58) and (59) we have: $\left[\rho\left(T_{\alpha}\right), \rho\left(T_{\beta}\right)\right]=\rho\left(\left[T_{\alpha}, T_{\beta}\right]\right)$.

Hence Equations (49) and (51) define the ECSM algebra $\tilde{\aleph}=\tilde{u}(2) \cong \mathbb{R}^{4}$ in terms of the SM algebra $\aleph=u(2) \cong \mathbb{R}^{4}$ as follows:

$$
\begin{aligned}
& {\left[\tilde{I}_{2}, \tilde{\tau}_{\alpha}\right]=\mathrm{F}\left(\theta_{s}\right)\left[I_{2}, \tau_{\alpha}\right]=\sin \theta_{s}\left[I_{2}, \tau_{\alpha}\right]=0,} \\
& {\left[\tilde{\tau}_{\alpha}, \tilde{\tau}_{\beta}\right]=\mathrm{F}\left(\theta_{s}\right)\left[\tau_{\alpha}, \tau_{\beta}\right]=\sin \theta_{s}\left[\tau_{\alpha}, \tau_{\beta}\right] .}
\end{aligned}
$$

\section{Properties}

The ECSM algebra is the $\mathrm{F}\left(\theta_{s}\right)$-valued SM algebra in $\mathrm{A}_{\mathrm{SM}}$. Consequently, we have the following properties for the $\tilde{\aleph}=\tilde{u}(2) \cong \mathbb{R}^{4}$ algebra:

\section{For angle $\theta_{s}=0$, we find an ECS trivial algebra \{0\} and the usual SM algebra.}

2. For angle $\theta_{s}= \pm \pi / 2$, we find the usual SM-algebra.

3. For every other value of angle $\theta$ s, we find many SM algebras that depend on ECS angle $\theta$ s. These are called ECSM algebras.

The terms $\left(\rho\left(I_{2}\right) \cdot \phi\right)$ and $\left(\rho\left(\tau_{\alpha}\right) \cdot \phi\right)$ in Equations (50) and (52) correspond to the Lie derivatives of $\phi$ with respect to $I_{2}$ and $\tau_{\alpha}$ :

$\mathfrak{J}_{\rho\left(I_{2}\right)} \phi=\sin \theta_{s}\left[\mathrm{I}_{2}, \phi\right]=0$, where $\left[\mathrm{I}_{2}, \phi\right]=0$,

$$
\mathfrak{I}_{\rho\left(\tau_{\alpha}\right)} \phi=\sin \theta_{s} \tau^{\alpha} \partial_{\alpha} \phi
$$

For a gentle smooth function on $M=S^{3} \times S^{1}$ that satisfies the eigenvalue equation $\partial_{\alpha} \phi=i m \phi$ with eigenvalues $(m=0, \pm 1, \pm 2, \ldots .$.$) , Equations (50) and (52) for the SM$ algebroid $\mathrm{A}_{\mathrm{SM}}$ become:

$\left[\mathrm{I}_{2}, \phi \tau_{\alpha}\right]=0$

$\left[\tau_{\alpha}, \phi \tau_{\beta}\right]=\phi\left[\tau_{\alpha}, \tau_{\beta}\right]\left(1+m \mathrm{~F}\left(\theta_{s}\right)\right)$, where $m=0, \pm 1, \pm 2, \ldots \ldots$

For $\left(\theta_{s}=0\right)$ or $(m=0)$, we find the usual SM-algebra. The other possibilities generated by the eigenvalues $m$ are under investigation for a further paper [58]. Following Mark Bugden, Peter Bouwkneg (2018)[59] and Cédric Fournel (2013)[44],when $A_{S M}$ is an SM algebroid, we can use the anchor map (Equation ()) to lift any vector bundle connection $\left(\nabla_{\mu}\right)$ on $\mathrm{A}_{\mathrm{SM}}$, to a socalled A connection $\left({ }^{A} \nabla_{\mu}\right)$ on $\mathrm{A}_{\mathrm{SM}}$ : 
${ }^{A} \nabla: \Gamma(A) \otimes \Gamma(A) \rightarrow \Gamma(A)$.

In this paper, we use a specific A connection, given by:

${ }^{A} \nabla_{\tilde{T}_{\alpha} \mu}\left(\mathrm{T}_{\beta}\right):=\nabla_{\rho\left(\mathrm{T}_{\alpha}\right) \mu}\left(T_{\beta}\right)=\rho\left(\mathrm{T}_{\alpha}\right) \nabla_{\mu}\left(T_{\beta}\right)$,

where $\nabla_{\mu}\left(T_{\beta}\right)$ is the bundle connection. Once we have an A connection, we can define the A curvature of ${ }^{A} \nabla_{\mu}$ as follows:

${ }^{A} \tilde{F}_{\gamma \mu v}:=\left[\nabla_{\rho\left(T_{\alpha}\right) \mu} \nabla_{\rho\left(T_{\beta}\right) v}\right]-\nabla_{\left[\rho\left(T_{\alpha}\right) v, \rho\left(T_{\beta}\right) v\right]}=\left[\rho\left(T_{\alpha}\right) \nabla_{\mu} \rho\left(T_{\beta}\right) \nabla_{v}\right]$
$-\left[\rho\left(T_{\alpha}\right), \rho\left(T_{\beta}\right)\right] \nabla_{\left[\mathrm{e}_{\mu}, \mathrm{e}_{v}\right]}=\rho\left(T_{\gamma}\right) \mathrm{F}_{\mu v}+\rho\left(T_{\gamma}\right) \nabla_{\mu} \nabla_{v}$

where

$F_{\mu \nu}=\left[\nabla_{\mu}, \nabla_{v}\right]-\nabla_{\left[\mathrm{e}_{\mu}, \mathrm{e}_{v}\right]}$,

is the curvature of the bundle connection $\nabla_{\mu}$.

\subsection{The ECSM-groupoid $\tilde{U}(2)$}

Following Marius Crainic and Rui Loja Fernandes (2003)[42], we now deduce the known inerrability.

1. Lie algebra bundles: For Lie algebroids with zero anchor map (Lie algebra bundles), the orbits are the points of M. Therefore, the conditions of the main theorem [42] are trivially satisfied, and we obtain the results of Douady and Lazard (1966) [60]:

Corollary 1 [42]: Any Lie algebra bundle is integrable to a Lie group bundle.

Corollary 2: The ECSM algebra is integrable to the ECSM group U(2) $)_{\mathrm{ECS}}$.

By Corollary 1, the SM algebra is integrable to a SM group. By the anchor of the SM algebroid $\mathrm{A}_{\mathrm{SM}}$ (Equations (53-54)), the SM and ECSM algebras are integrable to the SM group $\mathrm{U}(2)$ and ECSM group $\mathrm{U}(2)_{\mathrm{ECS}}$, respectively, up to the anchor homomorphic surjection between the SM and ECSM algebra.

From equations (29) and (53-54), we obtain the vector spaces of

$$
\sum_{\alpha} \varepsilon^{\alpha}(x) \tilde{T}_{\alpha}=\alpha(x) \tilde{I}_{2}+\beta(x)^{a} \tilde{\tau}_{a}, \alpha=(1,2,3,4)
$$

together with the commutator. Equations (70) and (60), (61) are referred to the ECSM algebra $\tilde{\aleph}=\tilde{u}(2) \cong \mathbb{R}^{4}$. We observe that:

$$
\begin{aligned}
& \alpha(x) \tilde{I}_{2}\left(\theta_{S}\right)+b(x) \cdot \tilde{\tau}\left(\theta_{S}\right)=\alpha(x) \mathrm{F}\left(\theta_{S}\right) I_{2}+b(x) \cdot F\left(\theta_{S}\right) \tau \\
& =F\left(\theta_{s}\right)\left(\begin{array}{cc}
\left(\alpha(x)+b_{3}(x)\right) & \left(b_{1}(x)-i b_{2}(x)\right) \\
\left(\alpha(x)+b_{3}(x)\right) & \left(\alpha(x)-b_{3}(x)\right)
\end{array}\right)=\tilde{\alpha}\left(x, \theta_{s}\right) I_{2}+\tilde{b}\left(x, \theta_{s}\right) \cdot \tau,
\end{aligned}
$$


with being the real arbitrary ECS parameters. These parameters are as follows:

$\tilde{a}\left(x, \theta_{s}\right)=a(x) \rho\left(\theta_{s}\right), \tilde{b}_{1}\left(x, \theta_{s}\right)=b_{1}(x) \rho\left(\theta_{s}\right)$,

$\tilde{b}_{2}\left(x, \theta_{s}\right)=b_{2}(x) \rho\left(\theta_{s}\right), \tilde{b}_{3}\left(x, \theta_{s}\right)=b_{3}(x) \rho\left(\theta_{s}\right)$,

Here, $\mathrm{F}\left(\theta_{s}\right)$ is the smooth function on $S^{3} \times S^{1}$, given by the Equation(55); $\theta_{s}$ is the ECS mixing angle, which is strictly a global parameter; and $a(x), b_{1}(x), b_{2}(x), b_{3}(x)$ are the real arbitrary group parameters of the local U(2) gauge group [56-57].

A representation of the $\mathrm{SM}$ gauge groupoid $\tilde{U}(2)$ on a vector bundle $\mathrm{E} \rightarrow \mathrm{M}$ induces a representation ${ }^{A} \nabla$ of $\mathrm{A}_{\mathrm{SM}}$ on $\mathrm{E} \rightarrow \mathrm{M}$, defined by the following system of partial differential equations (PDE):

$$
\nabla_{\tilde{\varepsilon}_{\alpha}} \tilde{U}\left(\tilde{\varepsilon}_{\alpha}\left(x, \theta_{s}\right)\right)=\left(\left.\frac{d}{d \tilde{\varepsilon}_{\alpha}} \tilde{U}\left(\tilde{\varepsilon}_{\alpha}\left(x, \theta_{s}\right)\right)\right|_{\tilde{\varepsilon}_{\alpha}=0}\right) \tilde{U}\left(\tilde{\varepsilon}_{\alpha}\left(x, \theta_{s}\right)\right)=i \mathrm{~T}^{\alpha} \tilde{U}\left(\tilde{\varepsilon}_{\alpha}\left(x, \theta_{s}\right)\right),
$$

where

$\left(\left.\frac{d}{d \tilde{\varepsilon}_{\alpha}} \tilde{U}\left(\tilde{\varepsilon}_{\alpha}\left(x, \theta_{s}\right)\right)\right|_{\tilde{\varepsilon}_{\alpha}=0}\right)=i \mathrm{~T}^{\alpha}, \alpha=(1,2,3,4)$

$\mathrm{T}^{\alpha}$ are the generators of the SM algebra, given by Equation (29). Using Equations (71)-(72), the solution of the PDE system is given by:

$\tilde{U}\left(\mathrm{x}, \theta_{S}\right)=\left\{\exp \left(i \tilde{\alpha}\left(x, \theta_{S}\right) I_{2}\right) \exp \left(i \tilde{b}\left(x, \theta_{S}\right) \cdot \tau\right) \mid \tilde{\alpha}\left(x, \theta_{S}\right) \in \mathbb{R}, \theta_{S} \in \mathbb{R}, \tilde{b}\left(x, \theta_{S}\right) \in \mathbb{R}^{3}\right\} \in \tilde{U}(2)$

with $\tilde{U}(2)$ being the SM gauge groupoid of two dimensions. Therefore, it follows that

$\tilde{U}\left(\mathrm{x}, \theta_{S}\right)=\left\{\exp \left(i \tilde{\alpha}\left(x, \theta_{s}\right)\right) \exp \left(i \tilde{b}\left(x, \theta_{s}\right) \cdot \tau\right) \mid \tilde{\alpha}\left(x, \theta_{s}\right) \in \mathbb{R}, \theta_{s} \in \mathbb{R}, \tilde{b}\left(x, \theta_{S}\right) \in \mathbb{R}^{3}\right\}$

$=\tilde{\mathrm{U}}(\mathrm{x})_{1} \tilde{\mathrm{U}}(\mathrm{x})_{2} \in \tilde{U}(1) \times S \tilde{U}(2)$

where

$\tilde{U}\left(\mathrm{x}, \theta_{s}\right)_{1}=\left\{\exp \left(i \tilde{\alpha}\left(x, \theta_{S}\right)\right) \mid \tilde{\alpha}\left(x, \theta_{S}\right), \in \mathbb{R}, \theta_{s} \in \mathbb{R}\right\} \in \tilde{U}(1)$,

$\tilde{U}(1)$ is the unitary gauge groupoid in one dimension, generated by phase $I_{2}$ [56-57], and $S \tilde{U}(2)$ is the special unitary gauge groupoid in two dimensions, generated by the 3-tuple $\tau_{\alpha}=\left(\tau_{1}, \tau_{2}, \tau_{3}\right)$ of the Hermitian and traceless matrices, respectively[56-57]. By comparing equations (76) and (77), we have:

$\tilde{U}(2)=\tilde{U}(1) \times S \tilde{U}(2)$.

For the Equations (71)-(72) and (75), we have:

$$
\begin{aligned}
& \tilde{U}\left(\mathrm{x}, \theta_{S}\right)=\left\{\exp \left(i F\left(\theta_{S}\right) \alpha(x) I_{2}\right)+\left(i F\left(\theta_{S}\right) b(x) \cdot \tau\right) \mid \alpha(x) \in \mathbb{R}, \theta_{S} \in \mathbb{R}, b(x) \in \mathbb{R}^{3}\right\}, \\
& =U(\mathrm{x})^{\mathrm{F}\left(\theta_{s}\right)} \in \tilde{U}(2)
\end{aligned}
$$

where $U(\mathrm{x}) \in U(2)[56-57]$,

$$
\begin{aligned}
& \tilde{U}\left(\mathrm{x}, \theta_{S}\right)=\left\{\exp \left(i F\left(\theta_{s}\right) \alpha(x)\right) \exp \left(i F\left(\theta_{s}\right) b(x) \cdot \tau\right) \mid \alpha(x) \in \mathbb{R}, \theta_{s} \in \mathbb{R}, b(x) \in \mathbb{R}^{3}\right\}, \\
& =\mathrm{U}(\mathrm{x})_{1}^{\mathrm{F}\left(\theta_{s}\right)} \mathrm{U}(\mathrm{x})_{2}^{\mathrm{F}\left(\theta_{s}\right)} \in \tilde{U}(1) \times S \tilde{U}(2)
\end{aligned}
$$

where

$$
\tilde{U}\left(\mathrm{x}, \theta_{s}\right)_{1}=\left\{\exp \left(i F\left(\theta_{S}\right) \alpha(x)\right) \mid \alpha(x), \in \mathbb{R}, \theta_{s} \in \mathbb{R}\right\}=U(\mathrm{x})_{1}^{\mathrm{F}\left(\theta_{s}\right)} \in \tilde{U}(1),
$$


$\tilde{U}\left(\mathrm{x}, \theta_{S}\right)_{2}=\left\{\exp \left(i F\left(\theta_{s}\right) b(x) \cdot \tau\right) \mid \theta_{s} \in \mathbb{R}, b(x) \in \mathbb{R}^{3}\right\}=U(\mathrm{x})_{2}^{\mathrm{F}\left(\theta_{s}\right)} \in S \tilde{U}(2)$,

and $U(\mathrm{x})_{1} \in U(1), U(\mathrm{x})_{2} \in S U(2)$ [56-57].

Therefore from equations (79), (80), we have:

$$
U(\mathrm{x})^{\mathrm{F}\left(\theta_{s}\right)}=\mathrm{U}(\mathrm{x})_{1}^{\mathrm{F}\left(\theta_{s}\right)} \mathrm{U}(\mathrm{x})_{2}^{\mathrm{F}\left(\theta_{s}\right)} \text {. }
$$

We observe that the $\tilde{U}(2), \mathrm{SU}(2)$, and $\tilde{U}(1)$ gauge groupoids are the $\mathrm{F}\left(\theta_{s}\right)$-valued $\mathrm{U}(2)$, $\mathrm{SU}(2)$, and $\mathrm{U}(1)$ gauge groups [56-57] in $\theta$ s.

\section{Properties}

The gauge groupoid $\tilde{U}(2)$, therefore, has the following properties:

1. For angle $\theta s=0$, we obtain the trivial group $\{1\}$.

2. For angle $\theta s= \pm \pi / 2$, we obtain the usual SM gauge group $U(2)$.

3. For every other value of angle $\theta$, we obtain many SM gauge groups $U(2)$ that depend on the ECS angle $\theta$ s; therefore, these groups are called the ECSM gauge group $U(2)_{E C S}$.

We regard the ECSM gauge group sectors as mirror SM models [61-63] that are suppressed for small values of the ECS angle $\theta_{s}$. These groups will be explored in future work [64].

The ECS-angle $\theta_{s}$ in the anchor map (Equation (53-54)) is strictly a global parameter, and may originate form a different group. In this case, the ECS angle $\theta_{s}$ could originate either from the global $\mathrm{SO}(3)_{\mathrm{ECS}}$ group or from the finite subgroups of the ECS Möbius transformations (see below). Furthermore, as Weinstein article illustrates [42], there is no assumption that a gauge transformation actually extends to the entire object $\mathrm{U}(2)$ : it may be that the gauge symmetry does not extend globally but affects only a part of $U(2)$, while the ECS symmetry extend globally.

\subsection{The Lagrangian of the gauge groupoid $\tilde{U}(2)$}

The A connection coefficient of the gauge groupoid $\tilde{U}(2)$ stems from the A connection:

$$
{ }^{A} \tilde{A}_{\mu \alpha \beta}(x)=\tilde{\mathrm{T}}_{\alpha}\left({ }^{A} \nabla_{\mu} \mathrm{T}_{\beta}\right)=\rho\left(\mathrm{T}_{\alpha}\right) \nabla_{\mu}\left(T_{\beta}\right)=\sin \theta_{s} \mathrm{~T}_{\alpha}\left(\nabla_{\mu} \mathrm{T}_{\beta}\right)=F\left(\theta_{s}\right) A_{\mu \alpha \beta}(\mathrm{x}),
$$

where

$$
A_{\alpha \beta \mu}(\mathrm{x})=\mathrm{T}_{\alpha}\left(\nabla_{\mu} \mathrm{T}_{\beta}\right)
$$

is the SM connection coefficient, and $F\left(\theta_{s}\right)$ is the smooth function on $S^{3} \times S^{1}$, given by Equation (55). The gauge covariant derivative is as follows:

$$
D_{\mu}\left(x, \theta_{s}\right)=\partial_{\mu}-i g \tilde{A}_{\mu}\left(x, \theta_{s}\right)=\partial_{\mu}-i g F\left(\theta_{s}\right) A_{\mu}(x) .
$$


The transformation of $A_{\mu}\left(x, \theta_{s}\right)$ is derived from the following equation:

$$
D_{\mu}^{\prime}\left(x, \theta_{s}\right)=\mathrm{e}^{i \varepsilon\left(x, \theta_{s}\right)} D_{\mu}\left(x, \theta_{s}\right) .
$$

After same matrix manipulations, the solution of Equation (87) for $\tilde{A}_{\mu}^{\prime}\left(x, \theta_{s}\right)$ in terms of $\tilde{A}_{\mu}\left(x, \theta_{s}\right)$ is:

$$
\tilde{A}_{\mu}^{\prime}\left(x, \theta_{s}\right)=\mathrm{e}^{i \varepsilon\left(x, \theta_{s}\right)} \tilde{A}_{\mu}\left(x, \theta_{s}\right) \mathrm{e}^{-i \varepsilon\left(x, \theta_{s}\right)}-\frac{1}{g} \partial_{\mu} \varepsilon\left(x, \theta_{s}\right),
$$

where $\mathrm{e}^{-i \varepsilon\left(x, \theta_{s}\right)}$ is the inverse of the matrix $\mathrm{e}^{i \varepsilon\left(x, \theta_{s}\right)}$. The strength of the ECSM and ordinary SM gauge fields $\tilde{A}_{\mu}^{\alpha}\left(x, \theta_{s}\right)$ and $A_{\mu}^{\alpha}(x)$ are defined as follows:

$$
\begin{aligned}
& \tilde{\mathrm{F}}_{\mu v}\left(x, \theta_{s}\right)=\frac{1}{-g}\left[D_{\mu}\left(x, \theta_{s}\right), D_{v}\left(x, \theta_{s}\right)\right]=\partial_{\mu} \tilde{A}_{v}\left(x, \theta_{s}\right)-\partial_{v} \tilde{A}_{\mu}\left(x, \theta_{s}\right) \\
& -i g\left[\tilde{A}_{\mu}\left(x, \theta_{s}\right), \tilde{A}_{v}\left(x, \theta_{s}\right)\right]=F\left(\theta_{s}\right)\left(\partial_{\mu} A_{v}(x)-\partial_{v} A_{\mu}(x)-i g F\left(\theta_{s}\right)\left[A_{\mu}(\mathrm{x}), A_{v}(x)\right]\right) . \\
& =F\left(\theta_{s}\right) \mathrm{F}_{\mu \nu}(x)
\end{aligned}
$$

Similarly, $\tilde{\mathrm{F}}_{\mu v}\left(x, \theta_{s}\right)$ and $\mathrm{F}_{\mu v}(x)$ can also be expressed as linear combinations of generators:

$$
\tilde{F}_{\mu v}\left(x, \theta_{s}\right)=\tilde{F}_{\mu \nu}^{\alpha}\left(x, \theta_{s}\right) \mathrm{T}_{\alpha}=F_{\mu \nu}^{\alpha}(x) \tilde{\mathrm{T}}_{\alpha}=F\left(\theta_{s}\right) \mathrm{F}_{\mu \nu}^{\alpha}(x) \mathrm{T}_{\alpha}=F\left(\theta_{s}\right) \mathrm{F}_{\mu \nu}(x) .
$$

The transformation law for the matrix $\tilde{F}_{\mu v}\left(x, \theta_{s}\right)$ is:

$$
\tilde{F}_{\mu \nu}^{\prime}\left(x, \theta_{s}\right)=\mathrm{e}^{i \varepsilon\left(x, \theta_{s}\right)} \tilde{F}_{\mu v}\left(x, \theta_{s}\right) \mathrm{e}^{-i \varepsilon\left(x, \theta_{s}\right)} .
$$

Thus, we can write down a kinetic energy term as follows:

$$
\mathfrak{I}_{E C S}=-\frac{1}{4} \tilde{F}_{\mu v}^{\alpha}\left(x, \theta_{s}\right) \tilde{F}_{\alpha}^{\mu v}\left(x, \theta_{s}\right) .
$$

The Lagrangian density of the ECSM is given by:

$$
\begin{aligned}
& \mathfrak{I}_{E C S M}\left(x, \theta_{s}\right)=-\bar{\psi}\left[\gamma^{\mu}\left(\partial_{\mu}-i g \tilde{A}_{\mu}^{\alpha}\left(x, \theta_{s}\right) T_{\alpha}\right)+m\right] \psi-\frac{1}{4} \tilde{\mathrm{F}}^{\alpha \mu v}\left(x, \theta_{s}\right) \tilde{\mathrm{F}}_{\alpha \mu v}\left(x, \theta_{s}\right) \\
& =-\bar{\psi}\left[\gamma^{\mu}\left(\partial_{\mu}-i g F\left(\theta_{s}\right) A_{\mu}^{\alpha}(x) T_{\alpha}\right)+m\right] \psi-\frac{\mathrm{F}\left(\theta_{s}\right)^{2}}{4} \mathrm{~F}^{\alpha \mu v}(\mathrm{x}) \mathrm{F}_{\alpha \mu v}(x)+\mathrm{O}\left(\rho\left(\theta_{s}\right)^{4}\right)
\end{aligned}
$$

We see that the Lagrangian is given in the initial form before the spontaneous breaking of the groupoid $\tilde{U}(2)$ symmetry. The $\tilde{U}(1)_{Y}$ ECS gauge groupoid is implicitly assumed in the second term of the summation over $S \tilde{U}(2)_{L}$. The first term represents the kinetic terms and the gauge interactions of fermions, provided by the covariant derivative(s).

\subsection{ECSM gauge field mass}


It turns out that the concept of spontaneous symmetry breaking plays an important role in the proposed theory of ECS electroweak interaction. The broken large groupoid symmetry

$$
S \tilde{U}(2)_{L} \times \tilde{U}(1)_{Y} \rightarrow \tilde{U}(1)_{E M}
$$

gives the massive ECS particles $\tilde{W}$ and $\tilde{Z}$ bosons. In equations (80) and (81), we observe that the spontaneous breaking of the large groupoid symmetry can only occur when the symmetry of electroweak interaction breaks spontaneously:

$\mathrm{SU}(2)_{\mathrm{L}} \times \mathrm{U}(1)_{\mathrm{Y}} \rightarrow \mathrm{U}(1)_{\mathrm{EM}}$

Equation (95) predicts the massive particles $\mathrm{W}$ and $\mathrm{Z}$ bosons, whose correct mass has already been known since 1983 [4-6]. In Equation (81), $\tilde{U}(1)_{E M}$ is the ECS gauge groupoid of electromagnetism, which is the $\mathrm{F}\left(\theta_{s}\right)$-valued $\mathrm{U}(1)_{\mathrm{EM}}$ common electromagnetism in $\theta s$. Therefore, we may view the ECS electromagnetism sectors of the $\tilde{U}(1)_{E M}$ gauge groupoid as the mirror sectors [61-63] that are suppressed for small ECS angles $\theta_{s}$ (a proposition to be explored in further work [64]). The most general Lagrangian consistent with the gauge invariance, Lorentz invariance, and renormalizability of the ECS gauge groupoid $\tilde{U}(1) \times S \tilde{U}(2)$ :

$$
\begin{aligned}
& \mathfrak{I}_{E C S}=-\frac{1}{2}\left|\left(\partial_{\mu}-i \tilde{A}_{\mu} \cdot t^{(\phi)}-i \tilde{B}_{\mu} y^{(\phi)}\right) \tilde{\phi}\right|-\frac{\mu^{2}}{2} \tilde{\phi}^{*} \tilde{\phi}-\frac{\lambda}{4}\left(\tilde{\phi}^{*} \tilde{\phi}\right)^{2} \\
& =-\frac{1}{2}|\sin | \theta_{s}\left|\left\{\partial_{\mu}-i \sin \left|\theta_{s}\right|\left(A_{\mu} \cdot t^{(\phi)}-i B_{\mu} y^{(\phi)}\right)\right\} \phi\right|-\frac{\mu^{2}}{2} \sin ^{2}\left|\theta_{s}\right| \phi^{*} \phi-\frac{\lambda}{4} \sin ^{4}\left|\theta_{s}\right|\left(\phi^{*} \phi\right)^{2}
\end{aligned}
$$

where

$$
\tilde{A}_{\mu}\left(x, \theta_{s}\right)=F\left(\theta_{s}\right) A_{\mu}(x), \tilde{B}_{\mu}\left(x, \theta_{s}\right)=F\left(\theta_{s}\right) \mathrm{B}_{\mu}(x), \tilde{\phi}\left(x, \theta_{s}\right)=F\left(\theta_{s}\right) \phi(x) .
$$

The scalar Lagrangian (96) then yields an ECSM vector boson mass term:

$$
\begin{aligned}
& \left.-\frac{1}{2}\left|\left(\partial_{\mu}-i \tilde{A}_{\mu}\left(\theta_{s}\right) \cdot \tau^{(\phi)}-i \tilde{B}_{\mu}\left(\theta_{s}\right) \cdot \tau^{(\phi)}\right) \tilde{\phi}\left(\theta_{s}\right)\right|^{2}=\frac{1}{2} \mid\left(\frac{g}{2} \tilde{A}_{\alpha \mu}\left(\theta_{s}\right) T^{\alpha}-\frac{g^{\prime}}{2} \tilde{A}_{\alpha \mu}\left(\theta_{s}\right)\right)\right)\left.\left(\begin{array}{l}
0 \\
\tilde{v}\left(\theta_{s}\right)
\end{array}\right)\right|^{2}, \\
& =\frac{\tilde{v}^{2}\left(\theta_{s}\right) g^{2}}{4} \tilde{W}_{\mu}^{*}\left(\theta_{s}\right) \tilde{W}^{\mu}\left(\theta_{s}\right)-\frac{\tilde{v}^{2}\left(x, \theta_{s}\right) g^{\prime 2}}{4} \tilde{Z}_{\mu}\left(\theta_{s}\right) \tilde{Z}^{\mu}\left(\theta_{s}\right)
\end{aligned}
$$

where $g$ and $g^{\prime}$ are the coupling constants. The ECSM masses are given as follows:

$$
M_{\tilde{A}}\left(\theta_{s}\right)=0, M_{\tilde{W}}\left(\theta_{s}\right)=\frac{1}{2} g \tilde{v}\left(\theta_{s}\right), M_{\tilde{Z}}\left(\theta_{s}\right)=\frac{1}{2} \tilde{v}\left(\theta_{s}\right) \sqrt{g^{2}+g^{\prime 2}} .
$$

Here, $\tilde{W}^{ \pm}$and $\tilde{Z}^{0}$ are the gauge bosons that mediate the ECS-exchanging electroweak interaction between the families of fermions (for details see [31-34]). Using Equation (97), the ECSM masses become

$$
M_{\tilde{A}}\left(\theta_{s}\right)=M_{A}=0, M_{\tilde{W}}\left(\theta_{s}\right)=M_{W} \mathrm{~F}\left(\theta_{s}\right), M_{\tilde{Z}}\left(\theta_{s}\right)=M_{Z} \mathrm{~F}\left(\theta_{s}\right),
$$


where

$$
M_{A}=0, M_{W}=\frac{1}{2} g v, M_{Z}=\frac{1}{2} v \sqrt{g^{2}+g^{\prime 2}}
$$

are the gauge boson masses of the SM vector [4-6]. The ECS Higgs potential in Equation (96) is given by

$$
V_{0}(\tilde{\phi})=\tilde{\mu}^{2}|\tilde{\phi}|^{2}+\lambda|\tilde{\phi}|^{4} .
$$

Decomposing into physical and Nambu-Goldstone (NG) modes, we notice that, when we expand about some general vacuum with $\varphi=v$ undetermined, the resulting masses are:

$$
\begin{aligned}
& m_{\tilde{H}}^{2}\left(\theta_{s}\right)=\tilde{\mu}^{2}+6 \lambda \tilde{v}\left(\theta_{s}\right)^{2}, \\
& m_{N G B}^{2}\left(\theta_{s}\right)=\tilde{\mu}^{2}+2 \lambda \tilde{v}\left(\theta_{s}\right)^{2},
\end{aligned}
$$

where $\mathrm{m}_{\mathrm{GB}}$ is the common mass of the $\mathrm{NG}$ bosons. At the minimum of the potential in Equation (), we obtain the bare ECS Higgs mass:

$$
m_{\tilde{H}}^{2}\left(\theta_{s}\right)=-2 \tilde{\mu}^{2}=4 \lambda \tilde{v}\left(\theta_{s}\right)^{2}=4 \lambda v^{2} F\left(\theta_{s}\right)^{2}=m_{H}^{2} F\left(\theta_{s}\right)^{2},
$$

where

$$
m_{H}^{2}=4 \lambda v^{2} .
$$

The SM Higgs gauge boson [4-6] and all the NG bosons are massless.

\subsection{Masses of the ECSM quarks and leptons}

The masses and mixing of the ECSM quarks and leptons have a common origin, as suggested in the SM [4-6]. They arise from the Yukawa interactions with the ECS Higgs condensate:

$$
L_{Y}=-Y_{i j}^{d} Q_{L i}^{I} \phi\left(F\left(\theta_{s}\right)\right) \bar{d}_{R j}^{I}-Y_{i j}^{u} Q_{L i}^{I} \varepsilon \phi^{*}\left(F\left(\theta_{s}\right)\right) \overline{\mathrm{u}}_{R j}^{I}+h . c
$$

where $Y_{i j}^{u, d}$ are $3 \times 3$ complex matrices, $\phi\left(F\left(\theta_{s}\right)\right)$ is the ECS Higgs field, i, j are generation labels, $\varepsilon$ is the $2 \times 2$ antisymmetric tensor, $Q_{L i}^{I}$ are left-handed ECSM quark doublets, and $\bar{d}_{R j}^{I}$ and $\overline{\mathrm{u}}_{R j}^{I}$ are, respectively, right-handed down- and up-type ECS quark singlets in the weak eigenstate.

When $\tilde{\phi}\left(\theta_{s}\right)$ acquires a vacuum expectation value, $\left(\left\langle\tilde{\phi}\left(\theta_{s}\right)\right\rangle=\left(0, \tilde{v}\left(\theta_{s}\right) / \sqrt{2}\right)\right)$, Equation (107) yields mass terms for the ECSM quarks, as follows:

$$
L_{Y}=-Q_{L i}^{I} m_{i j}^{\tilde{d}}\left(\theta_{s}\right) \bar{d}_{R j}^{I}-Y_{i j}^{u} Q_{L i}^{I} m_{i j}^{\tilde{u}}\left(\theta_{s}\right) \overline{\mathrm{u}}_{R j}^{I}+h . c,
$$

where 


$$
\begin{aligned}
& m_{i j}^{\tilde{d}}\left(\theta_{s}\right)=\sum_{n} Y_{i j}^{\tilde{d}}\left\langle\tilde{\phi}^{0}\left(\theta_{s}\right)\right\rangle=m_{i j}^{d} \mathrm{~F}\left(\theta_{s}\right), m_{i j}^{\tilde{u}}\left(\theta_{s}\right)=\sum_{n} Y_{i j}^{\tilde{u}}\left\langle\tilde{\phi}^{0}\left(\theta_{s}\right)\right\rangle=m_{i j}^{u} \mathrm{~F}\left(\theta_{s}\right) \\
& m_{i j}^{d}=\sum_{n} Y_{i j}^{d}\left\langle\phi^{0}\right\rangle, m_{i j}^{u}=\sum_{n} Y_{i j}^{u}\left\langle\phi^{0}\right\rangle
\end{aligned}
$$

are the SM quark masses [4-6]. The ECSM quark masses depend on the arbitrary couplings and cannot be predicted. Furthermore, since ECSM quarks are not observed in isolation, their masses are not precisely defined. Similarly, for ECSM leptons, we have:

$$
m_{i j}^{\tilde{l}}\left(\theta_{s}\right)=\sum_{n} Y_{i j}^{\tilde{l}}\left\langle\tilde{\phi}^{0}\left(\theta_{s}\right)\right\rangle=m_{i j}^{l} \mathrm{~F}\left(\theta_{s}\right)
$$

where

$$
m_{i j}^{l}=\sum_{n} Y_{i j}^{l}\left\langle\phi^{0}\right\rangle
$$

are the SM lepton masses [4-6].

\section{Finite subgroups of the ECS Möbius transformations}

We observe that the $\tilde{U}(2), \mathrm{SU}(2)$, and the $\tilde{U}(1)$ ECS gauge groupoid are the $\mathrm{F}\left(\theta_{s}\right)$-valued $\mathrm{U}(2), \mathrm{SU}(2)$, and $\mathrm{U}(1)$ gauge groups [4-6] in $\theta s$. The ECS angle $\theta_{s}$ in Equation (55) is strictly an arbitrary global parameter, and may originate form a different group - either the $\mathrm{SO}(3)_{\mathrm{ECS}}$ group or from the finite subgroups of the ECS Möbius transformations as we explain in this section.

Let $\Gamma$ be an ECS subgroup of $\mathrm{PSL}_{2}(\mathrm{C})_{\mathrm{ECS}}$, consisting of elliptic elements together with the identity. Then $\Gamma_{\mathrm{ECS}}$ is conjugate in $\mathrm{PSL}_{2}(\mathrm{C})_{\mathrm{ECS}}$ to a subgroup of $\mathrm{PSU}_{2}(\mathrm{C})_{\mathrm{ECS}}$ [53-54].

Now, by the group isomorphism (12), for every finite subgroup of ECS rotations (i.e. a subgroup of $\mathrm{PSU}_{2}(\mathrm{C})_{\mathrm{ECS}}$ ), we have the following:

For a given finite group, $\Gamma_{\mathrm{ECS}}$, of ECS rotations in $\mathrm{C}^{\infty}$ (Equation ()), one of the following holds [53-54]:

\section{1. $\Gamma$ is ECS-cyclic;}

\section{2. . is ECS-dihedral;}

3. $\Gamma$ is the ECS symmetry group of a regular ECS tetrahedron $\left(A_{4}\right)$, ECS octahedron $\left(S_{4}\right)$, or ECS icosahedron $\left(A_{5}\right)$.

One can show that two finite ECS subgroups in $\mathrm{PSL}_{2}(\mathrm{C})_{\mathrm{ECS}}$ are conjugate if and only if they are isomorphic.

\subsection{ECS-dihedral group}

Here, $\mathrm{D}_{F}$ (with $\mathrm{F}$ being the number of fermions) refers to the symmetries of the Fermionic polygon (F-gon:having $F$ fermionicsides) - a group of order $2 F$. In abstract algebra, $\mathrm{D}_{2 F}$ refers to this same ECS dihedral group (for details of the Dirac equation on the polygon regions see [69-75]). $\mathrm{D}_{F}$ is a subgroup of $O(2)_{E C S} \cong U(1)_{E C S}$, i.e., the group of ECS rotations (about the origin) and ECS reflections (across axes through the origin) of the plane. However, the notation ' $\mathrm{D}_{F}$ ' is also used for a subgroup of $S O(3)_{E C S} \cong S U(2)_{E C S}$, which is also an abstract 
group: the proper symmetry group of an F-gon embedded in three-dimensional internal space (if the number of fermions is $\mathrm{F} \geq 3$ ).

\subsection{The averages of $F(\theta s)$ function over the triangulation of a convex fermionic F-gon}

The sum of the interior ECS angles of a simple $F$-gon is $(F-2) \pi$ radians. This is because any simple $F$-gon can be considered to be made up of $(F-2)$ triangles, each of which has an angle sum of $\pi$ radians:

$\theta_{s}(F)=\left(1-\frac{2}{F}\right) \pi$

where

$F=F_{\text {ordinary }}+F_{E C S}$

$F$ is the total number of fermions, given by the sum of ordinary $F_{\text {ordinary }}$ and ECS fermions $F_{E C S}$. Following [76], we find that the number $\left(\mathrm{C}_{\mathrm{F}}\right)$ of triangulations of a convex fermionic $F$-gon in the internal space satisfies the recursive formula

$$
C_{F}=C_{0} C_{F-1}+C_{1} C_{F-2}+\ldots+C_{F-1} C_{0} \text {, }
$$

where $\mathrm{C}_{0}=1$ [77-80]. The numbers $\mathrm{C}_{\mathrm{F}}$ are now called fermionic Catalan numbers. From (206), it follows that $\mathrm{C}_{1}=1, \mathrm{C}_{2}=2, \mathrm{C}_{3}=5$, and so on. Using generating functions and Segner's formula, an explicit formula for $\mathrm{C}_{\mathrm{F}}$ can be developed [80]:

$$
C_{F}=\frac{(2 F) !}{(F+1) ! F !},
$$

with $\mathrm{F}$ being the total number of fermions.

After the triangulation of a convex fermionic $F$-gon in three-dimensional internal space, Equation (55) becomes:

$\sin \left(\theta_{s}(\mathrm{~F})\right)_{\left(\Delta_{F}\right)} \equiv \frac{\pi \sin \left(\theta_{s}(\mathrm{~F})\right)_{\text {averge }}}{C_{F}}=\frac{2}{C_{F}}$, on $[0, \pi / 2]$,

where

$\sin \left(\theta_{s}(\mathrm{~F})\right)_{\text {averge }}=\frac{2}{\pi}$, on $[0, \pi / 2]$.

This is the normalised average sin of the ECS angle $\theta_{s}$ over the triangulation of a convex fermionic $F$-gon by the fermionic Catalan numbers $\mathrm{C}_{\mathrm{F}}$.

\section{Results}

Now that the averages of the $F\left(\theta_{s}\right)$ function have been determined by the fermionic Catalan numbers $\mathrm{C}_{\mathrm{F}}$, we consider two possible scenarios of ECS contribution to the SM: 
Loop level: The ECS Physics at loop-level differs from the SM physics; the ECSM mass is not identical to the SM mass; therefore,

$$
\sin \left(\theta_{S}(\mathrm{~F})\right)_{\left(\Delta_{n}\right)} \equiv \frac{2}{C_{n}}=\frac{M_{\tilde{\mathrm{p}}-\left(\Delta_{n}\right)}}{M_{p-S M}} \neq 1, \text { on }[0, \pi / 2]
$$

implies

$$
M_{\tilde{\mathrm{p}}-\left(\Delta_{n}\right)}=\sin \left(\theta_{S}(\mathrm{~F})\right)_{\left(\Delta_{n}\right)} M_{p-S M}=\frac{2 M_{p-S M}}{C_{F}} \text {, on }[0, \pi / 2],
$$

where $M_{\tilde{\mathrm{p}}-\left(\Delta_{2}\right)}$ are the triangulation masses of the ECS particles (from equation (208)), $M_{p-S M}$ are the corresponding masses of the SM particles, and $\mathrm{C}_{\mathrm{F}}$ are the fermionic Catalan numbers for the triangulations of the fermionic F-gon.

Tree level: The ECS Physics at tree level is the same as the SM physics, and the ECSM masses are identical to the SM masses. Therefore,

$$
\sin \left(\theta_{s}(\mathrm{~F})\right)_{\left(\Delta_{2}\right)} \equiv \frac{2}{C_{2}}=\frac{M_{\tilde{\mathrm{p}}-\left(\Delta_{2}\right)}}{M_{p-S M}}=1, \text { on }[0, \pi / 2]
$$

implies

$$
M_{p-S M}=M_{\tilde{\mathrm{p}}-\left(\Delta_{2}\right)},
$$

where $M_{\tilde{\mathrm{p}}-\left(\Delta_{2}\right)}$ are the ECS particle triangulation masses of the fermionic 2-gon, are the corresponding SM particles masses, and $\mathrm{C}_{2}=2$ is the fermionic Catalan number for the triangulation of the fermionic 2-gon.

In the proposed ECS model $((211),(212))$, the tree-level mass term, $\mu_{0}$, which sets the weak scale, is naturally $\sim \mathrm{m}_{\mathrm{w}}$. We thus only consider the effect of the new ECS physics at loop level.

Considering the one-loop contribution to the effective potential (102), the radiative corrections take the form:

$$
V_{1}(\tilde{\phi})=\frac{1}{64 \pi^{2}} \int d^{4} k S \operatorname{Tr} \log \left(k^{2}+M^{2}\left(\tilde{\phi}\left(\Delta_{F}\right)\right)\right)=\frac{\Lambda_{c}^{2}}{32 \pi^{2} v^{2}} \operatorname{STr} M\left(\tilde{\phi}\left(\Delta_{F}\right)\right)+\ldots
$$

where $S \operatorname{Tr}=\operatorname{Tr}(-1)^{F}$ defines the supertrace. This new contribution can be absorbed into $\mathrm{V}_{0}$ (Equation (102)) by shifting the bare $\mu^{2}$ :

$$
\begin{aligned}
& M_{\tilde{H}}^{2}=\mu_{0}^{2}+\frac{3 \Lambda_{C}^{2}}{32 \pi^{2} v^{2}} \operatorname{STrM}^{2}\left(\tilde{\phi}\left(\Delta_{F_{A}}\right)\right) \\
& =\mu_{0}^{2}+\frac{3 \Lambda_{C}^{2}}{32 \pi^{2} v^{2}}\left[M_{\tilde{H}}^{2}\left(\Delta_{F_{A}}\right)+2 M_{\tilde{W}}^{2}\left(\Delta_{F_{A}}\right)+M_{\tilde{Z}}^{2}\left(\Delta_{F_{A}}\right)-4 m_{\tilde{t}}^{2}\left(\Delta_{F_{A}}\right)\right]+\ldots
\end{aligned},
$$

The trace over the $\varphi$-dependent triangulations masses is given by

$$
\begin{aligned}
& \sum_{\text {scalars }} M^{2}\left(\tilde{\phi}\left(\Delta_{F}\right)\right)=\left(\left.\mathrm{m}_{\tilde{H}}^{2}\left(\Delta_{F}\right)\right|_{\tilde{\phi}=\nu}-\left.\mathrm{m}_{\tilde{H}}^{2}\left(\Delta_{F}\right)\right|_{\tilde{\phi}=0)}\right) \\
& +3\left(\left.\mathrm{~m}_{N G B}^{2}\right|_{\tilde{\phi}=\nu}-\left.\mathrm{m}_{N G B}^{2}\right|_{\tilde{\phi}=0)}\right)=\left.3 \mathrm{~m}_{\tilde{H}}^{2}\left(\Delta_{F}\right)\right|_{\tilde{\phi}=\nu}
\end{aligned} .
$$

Thus Equation (215) is reproducing. 
Here, $M_{\tilde{H}}\left(\Delta_{F}\right), M_{\tilde{W}, \tilde{Z}}\left(\Delta_{F}\right)$, and $m_{\tilde{t}}\left(\Delta_{F}\right)$ are the triangulation masses of the ECS top quark (from equations (211) and (3)), ECS $\tilde{W}, \tilde{Z}$, and ECS Higgs bosons ( $\tilde{H}$ ), respectively, and $v$ is the vacuum expectation value of the Higgs potential in the SM [4-6]. The dots include logarithmic corrections in $\Lambda_{C}$, as well as contributions independent of $\Lambda_{C}$ in the large $\Lambda_{C}$ limit. Using equations (211) and (3), we obtain the one-loop radiative corrections to the bare Higgs mass:

$M_{\tilde{H}}^{2}=\mu_{0}^{2}-\frac{3 m_{\tilde{t}}^{2}\left(\Delta_{F_{A}}\right) \Lambda_{C}^{2}}{8 \pi^{2} v^{2}}=\mu_{0}^{2}-\frac{3 \lambda_{t}^{2} \Lambda_{C}^{2}}{4 \pi^{2} C_{F}^{2}}$.

These corrections depend on the fermionic Catalan number $\mathrm{C}_{\mathrm{F}}$ (Equation.(207)), the top quark Yukawa coupling $\lambda_{t}$ and the cut-off energy scale $\Lambda_{C}$.

Following equations [9], an all-orders result for $M_{\tilde{H}}^{2}$ is given by the following equation:

$$
M_{\tilde{H}}^{2}=\mu_{0}^{2}+\Lambda_{c}^{2} \sum_{n=0}^{\infty} \tilde{c}_{n}(\lambda) \log ^{n}\left(\frac{\Lambda_{c}}{Q}\right),
$$

where $c_{0}=(32 \pi 2)^{-1} \mathrm{~S} \operatorname{Tr} \mathrm{M}^{2} / \mathrm{v}^{2}$. The remaining $c_{n}$ can be calculated recursively by the following relation:

$$
(1+n) c_{n+1}=\frac{d c_{n}}{d(\log Q)}=\beta_{i} \frac{\partial c_{n}}{\partial \lambda_{i}},
$$

where $\mu_{0}^{2}$ should ne independent of the renormalization scale Q. For example:

$$
\begin{aligned}
& \left(16 \pi^{2}\right) c_{1}=\frac{\lambda}{C_{F}^{2}}\left(144 \lambda-54 g^{2}-18 g^{\prime 2}+72 \lambda_{t}^{2}\right)+\frac{\lambda_{t}^{2}}{C_{F}^{2}}\left(27 \mathrm{~g}^{2}+17 g^{\prime 2}+96 \lambda^{2}-90 \lambda_{t}^{2}\right) \\
& -\frac{15}{2 C_{F}^{2}} g^{4}+\frac{25}{2 C_{F}^{2}} g^{\prime 4}+\frac{9}{2 C_{F}^{2}} g^{2} g^{\prime 2}
\end{aligned} .
$$

Since each order in $n$ involves more factors of $\left(16 \pi^{2} \mathrm{C}_{\mathrm{F}}^{2}\right)^{-1}$, we expect that, for large fermionic Catalan numbers $\mathrm{C}_{\mathrm{F}}$, the higher-loop contributions are unimportant. Our approach to the higher-order contributions yields that each $c_{n} \neq 0$ is suppressed by the large fermionic Catalan number $\mathrm{C}_{\mathrm{F}}$ for all $\mathrm{n}$ separately. In the infinite cutoff limit (or in a cutoff-independent solution), this would be the correct procedure for solving the fine-tuning problem [29]. Given that all $c_{n} \neq 0$ are independent of, and suppressed by, the large $\mathrm{C}_{\mathrm{F}}$ over the $\left(\lambda_{\mathrm{i}}\right)$ parameter, a solution exists. Therefore, our approach makes it possible to solve the fine-tuning problem, since an all orders of solutions exist. For an equal number of ordinary and ECS fermions, the calculated one-loop radiative corrections to the bare Higgs mass $\mu^{2}$ is given in Table 3 . 


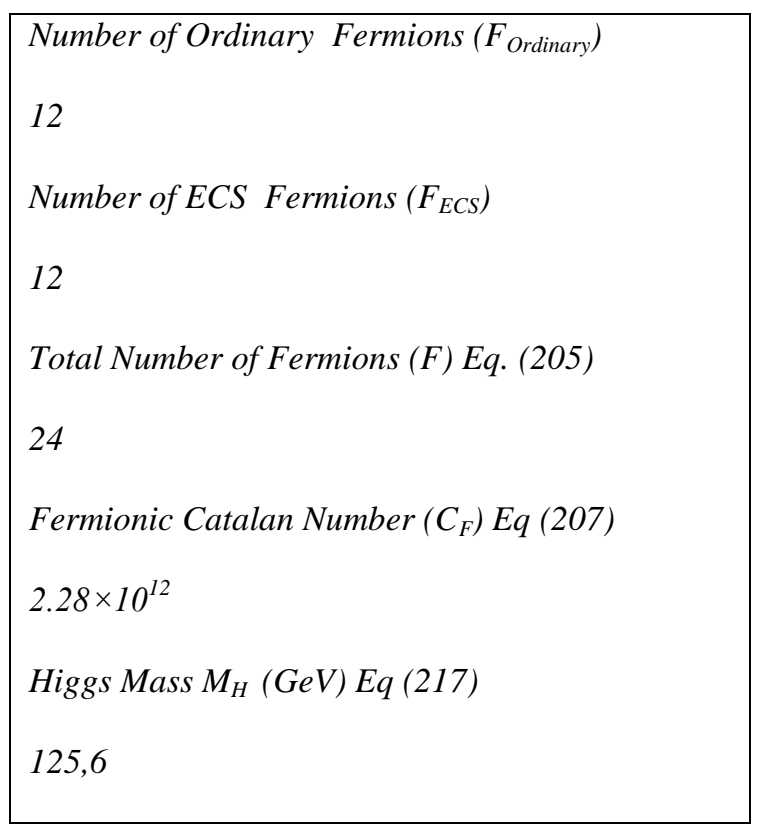

Table 3. One-loop radiative corrections to the bare Higgs mass, as calculated from Equation (217). For fermionic Catalan number $\mathrm{C}_{24}=2.28 \times 10^{12}$ (Equation (207)), and top quark Yukawa coupling $\lambda_{t}=0,93$ and the cut-off energy scale $\Lambda_{C .}=\mathrm{O}\left(10^{15} \mathrm{GeV}\right)$.

This result is very close to the experimental value of the Higgs mass [10,11]. The all-orders result for $M_{\tilde{H}}^{2}$ with a fermionic Catalan number $C_{24}$ becomes:

$M_{\tilde{H}}^{2}=\mu_{0}^{2}+\left(\frac{\Lambda_{c}}{C_{24}}\right)^{2} \sum_{n=0}^{\infty} c_{n}(\lambda) \log ^{n}\left(\frac{\Lambda_{c}}{Q}\right)$.

For instance, the calculated result

$$
\begin{aligned}
& \left(16 \pi^{2}\right) c_{1}=\frac{\lambda}{C_{24}^{2}}\left(144 \lambda-54 g^{2}-18 g^{\prime 2}+72 \lambda_{t}^{2}\right)+\frac{\lambda_{t}^{2}}{C_{24}^{2}}\left(27 \mathrm{~g}^{2}+17 g^{\prime 2}+96 \lambda^{2}-90 \lambda_{t}^{2}\right) \\
& -\frac{15}{2 C_{24}^{2}} g^{4}+\frac{25}{2 C_{24}^{2}} g^{\prime 4}+\frac{9}{2 C_{24}^{2}} g^{2} g^{\prime 2} \approx O\left(\frac{1}{C_{24}^{2}}\right)
\end{aligned}
$$

is of the order of the inverse square of the fermionic Catalan number of the 24F-gon. Our approach, therefore, solves the fine-tuning problem, since it provides an all-orders solution which is suppressed by the fermionic Catalan number $\mathrm{C}_{\mathrm{F}}$ for all $\mathrm{n}$ separately. For 24 fermions, we calculate a Higgs mass of $125,6 \mathrm{GeV}$ which is very close to the experimental value $[10,11]$.

\section{Conclusion}

Taking the SU(2) group of weak interactions in the presence of electric charge swap (ECS) symmetry as a starting point, we show that ordinary and non-regular (ECS) leptons are related by the ECS rotational $\mathrm{SO}(3)$ group. 
We investigate a version of the SM algebroid with the anchor map depending on the ECS angle $\theta s$. We find that many SM algebras depend on the ECS angle $\theta$ s. We call these ECSM algebras. Furthermore, the SM algebroid is integrable to the SM groupoid; so, our results potentially extend well beyond this case. Then, we investigate how the breaking of the SM groupoid symmetry gives the massive ECS particle. We find that the ECS particle mass is related to the SM particle mass through the ECS angle $\theta$ s.

We investigate the finite subgroups of the ECS Möbius transformations. In this case, the ECS-angle $\theta_{s}$ could originate from the ECS dihedral group that refers to the symmetry of the Fermionic polygon (F-gon). The ECS angle $\theta$ s can then be determined through the triangulation of a convex fermionic F-gon.

Finally, we find that, at loop-level, the ECS Physics is different from the SM physics, and the ECSM mass is suppressed by the fermionic Catalan numbers $C_{\mathrm{F}}$. For 24-fermions, the calculated one-loop radiative correction to the bare Higgs mass $\mu^{2}$ is $125,6 \mathrm{GeV}$-very close to the experimental value.

\section{References}

[1].M.E.Peskin ,D.V.Schorder An Introduction to Quantum Field Theory 1995 Preseus Book Publishing L.L.C Massachusetts U.S.A.

[2] Georgi H., Glashow.S.L, Phys.Rev.Lett. 32 (1974) 451. Mohapatra R. N., Unification and supersymmetry. The frontiers of quark-leptons physics Springer - Verlag (1986),

[3].G.G. Ross, Grand Unification Theories, Benjamin/Cummings (1985).Lucas V. and Raby. S, "Nucleon Decay in a Realistic SO(10) SUSY Gut," Phys. Rev. D 55 (1997).

[4].S.Weinberg, Phys. Rev. D 5 (1972) 1412.

[5].A. Salam, In Elementary Particle Theory: Relativistic Groups and Analyticity Nobel Symposium, ed. N. Svartholm, Vol. 8, Almqvist and Wiksell, Stockholm (1968).

[6].S.Glashow, Nucl. Phys. 22 (1961) 579. E.S. Abers; B.W. Lee (1973). "Gauge theories". Physics Reports. 9 (1): 1-141.M. Baak; et al. (2012). "The Electroweak Fit of the Standard Model after the Discovery of a New Boson at the LHC". The European Physical Journal C. 72 (11): 2205.

[7].C. Grojean, Philos Trans A Math Phys Eng Sci. 2015 Jan 13; 373(2032): 20140042.

[8].P.Grange, J.Mathiot, B.Mutet, E.Werner. Aspects of fine-tuning of the Higgs mass within finite field theories. Phys. Rev. D, 2013, 88 (12), pp.125015.

[9].C.Kolda ,H.Murayama, hep-ph/0003170 (March 2000).

[10].G.Aad et al. [ATLAS Collaboration], "Observation of a new particle in the search for the Standard Model Higgs boson with the ATLAS detector at the LHC," Phys. Lett. B 716, 1 (2012) [arXiv:1207.7214 [hep-ex]];

[11].S.Chatrchyan et al. [CMS Collaboration], "Observation of a new boson at a mass of $125 \mathrm{GeV}$ with the CMS experiment at the LHC,” Phys. Lett. B 716, 30 (2012) [arXiv:1207.7235 [hep-ex]]. 
[12].Howard Baer; Xerxes Tata (2006). "8 - The Minimal Supersymmetric Standard Model". Weak Scale Supersymmetry From Superfields to Scattering Events. Cambridge: Cambridge University Press. p. 127. ISBN 9780511617270.

[13].N.Arkani-Hamed; S. Dimopoulos; G. Dvali (1998). "The Hierarchy problem and new dimensions at a millimeter". Physics Letters. B429 (3-4): 263-272.N. Arkani-Hamed; S. Dimopoulos; G. Dvali (1999). ". Physical Review. D59 (8).

[14].Agashe K, Contino R, Pomarol A. 2005. The minimal composite Higgs model. Nucl. Phys. B 719, 165-187.(10.1016/j.nuclphysb.2005.04.035).Contino R, Da Rold L, Pomarol A. 2007. Light custodians in natural composite Higgs models. Phys. Rev. D 75, 055014.Gripaios B, Pomarol A, Riva F, Serra J. 2009.

[16].A.B. Arbuzov,Quantum Field Theory and the Electroweak Standard Model, Lectures given at the European School of High-Energy Physics (ESHEP), September 2017, Evora, Portugal , arXiv:1801.05670v1 [hep-ph].

[17]. K.A. Meissner, H. Nicolai, Phys.Lett.B648:312-317, 2007. Gustavo Marques Tavares,et.al, Higgs mass naturalness and scale invariance in the UV, arXiv:1308.0025v2 [hep-ph] 20 Aug 2013.

[18].Ralf Hempfling, The Next-to-Minimal Coleman-Weinberg Model, Phys.Lett. B379 (1996) 153158

[19].Arindam Das,et.al, Electroweak vacuum stability in classically conformal B - L extension of the standard model, Eur. Phys. J. C (2017) 77:122

[20].Nima Arkani-Hame,et.al, N-naturalness, arXiv:1607.06821v2 [hep-ph] (May 2017).

[21].Conway, J.H.; Sloane, N.J.A. (1999), Sphere packings, lattices and groups, Grundlehren der Mathematischen Wissenschaften, 290, With contributions by Bannai, E.; Borcherds, R. E.; Leech, J.; Norton, S. P.; Odlyzko, A. M.; Parker, R. A.; Queen, L.; Venkov, B. B. (Third ed.), New York, NY: Springer-Verlag, ISBN 978-0-387-98585-5,Cohn, Henry; Kumar, Abhinav (2009), "Optimality and uniqueness of the Leech lattice among lattices", Annals of Mathematics, 170 (3): 1003-1050,

[22].Leon,Jeffrey S.; Sims, Charles C. (1977). "The existence and uniqueness of a simple group generated by $\{3,4\}$-transpositions". Bull. Amer. Math. Soc. 83 (5): 1039-1040. Ronan, Mark (2006). Symmetry and the monster. Oxford University Press. pp. 178-179. ISBN 0-19-280722-6.

[23].Fowlie, Andrew; Balazs, Csaba; White, Graham; Marzola, Luca; Raidal, Martti (2016). "Naturalness of the relaxion mechanism". Journal of High Energy Physics. 2016 (8): 100.

[24].Fowlie, Andrew (2014). "CMSSM, naturalness and the? fine-tuning price? of the Very Large Hadron Collider". Physical Review D. 90(1): 015010.

[25].Fowlie, Andrew (2014). "Is the CNMSSM more credible than the CMSSM?". The European Physical Journal C. 74 (10).

[26].Austri, Roberto Ruiz de; Marzola, Luca; Raidal, Martti (2009). "Bayesian approach and naturalness in MSSM analyses for the LHC". Journal of High Energy Physics. 2009 (3): 075.

[27].Fichet, S. (2012). "Quantified naturalness from Bayesian statistics". Physical Review D. 86 (12): 125029.

[28].J.Rosaler, R.Harlander, Naturalness, Wilsonian Renormalization, and "Fundamental Parameters" in Quantum Field Theory (March 1, 2018), the epistemology of the Large Hadron Collider (LHC). 
[29].C.Wetterich, Fine-tuning problem and the renormalization group, Physics Letters B Vol 140, 3-4, 7 1984, 215-222.

[30].E.Bianchi,C.Rovelli, Why all these prejudices against a constant? (2010) arXiv:1002.3966 [astroph.CO].

[31].E.Koorambas, Lepton Electric Charge Swap at the $10 \mathrm{TeV}$ Energy Scale, Commun. Theor. Phys. 60 (2013) 561-570

[32].E.Koorambas, Quark-Quark of Swap Electric Charge Bound State Int. J. HEP, 2:4-1. (2015)

[33].E.Koorambas, Weak Space-Time Curvature Effects for Lepton Electric Charge Swap at High Energy Scale. Journal of Magnetohydrodynamics, Plasma, and Space Research , NOVA SCIENCE PUBLISHERS, INC, (2015), 20 (3), pp.333-354. 〈hal-01205635〉.

[34].E.Koorambas,THE ORIGIN OF SU (5) SYMMETRY BY LEPTON ELECTRIC CHARGE SWAP QUATERNIONS ALGEBRA. Christopher M. Villegas. Leptons: Classes, Properties and Interactions, Nova Science Publishers, Inc, pp.27-55, (2019), 978-1-53614-929-6. 〈hal-02067199〉.

[35].A-Wollmann Kleinert, F. Bulnes. Journal on Photonics and Spintronics, 2 (1) (2013).

[36].A.M. Polykov. Phys.Lett.B.103 (1981).R.J. Szabo. arXiv:hepth/0207142 (2002).

[37].A. Giveon, D. Kutasov. Reviews of Modern Physics, Vol. 71,No. 4 (1999).

[38].J. Polchinski. Phys. Rev. Lett. 75, (1995) 4724.

[39].J. Polchinski. arXiv: hep-th/9611050 (1996).

[40].Awodey, Steve (2010) [2006]. Category Theory. Oxford Logic Guides. 49 (2nd ed.). Oxford University Press. ISBN 978-0-19-923718-0. Geroch, Robert (1985). Mathematical physics ([Repr.] ed.). University of Chicago Press. pp. 7. ISBN 978-0-226-28862-8.Rosen, Robert (1958). "The representation of biological systems from the standpoint of the theory of categories" . Bulletin of Mathematical Biophysics. 20 (4): 317-341.

[41].M.Crainic,R.L.Fernandes, Integrability of Lie brackets, Annals of Mathematics, 157 (2003), 575620 .

[42].A.Weinstein,Groupoids:Unifying Internaland External Symmetry, NOTICES OF THE AMS, VOLUME 43, NUMBER 7 (JULY 1996), 744-752.

[43].J.C.Morton,J.Vicary,2012 The Categorified Heisenberg Algebra I: A Combinatorial Representation arXiv:1207.2054 [math.QA].

[44].Cédric Fournel. Gauge theories and generalized connections on transitive Lie algebroids. Mathematical Physics [math-ph]. Aix-Marseille Université, 2013. English. tel-01263377

[45]. M. F. Atiyah. Vector bundles over an elliptic curve. Proc. London Maths. Soc., 7:414-452, 1957.

[46].S.Lazzarini and T. Masson. Connections on Lie algebroids and on derivationbased noncommutative geometry. J. Geom. Phys., 62:387-402, 2012.

[47].S.Lazzarini and T. Masson. Connections on Lie algebroids and on derivationbased noncommutative geometry. Journal of Geometry and Physics, 62:387-402, 2012.

[48].Christopher M. Hull JHEP07(2007)080,Chris Hull and Barton Zwiebach JHEP09(2009)099I. 
[49].Vaisman,J. Math. Phys. 54, 123507 (2013), H.Mori, S.Sasaki,K.Shiozawa, J. Math. Phys. 61, 013505 (2020), H.Mori, S.Sasaki J. Math. Phys. 61, 123504 (2020)

[50].F.Halzen and A.D. Martin, Quarks and Leptons: An Introduction Course in Modern Particle Physics, John Wiley \& Son, New York (1984) 251-252, 292-301.

[51].G. Fubini, Atti Istit. Veneto 63 (1904) 502.

[52].E. Study, Math. Ann. 60 (1905) 321.

[53].Ulrich Pinkall, Differential Geometry II (Analysis and geometry on manifolds) Lecture notes, (July 10, 2019), Technische Universität Berlin Institut für Mathematik.

[54].Ralph L. Cohen ,The Topology of Fiber Bundles, Lecture Notes, Dept. of Mathematics Stanford University, (August, 1998).

[55].Hajime TANIGUCHI, A Note on the Differential of the Exponential Map and Jacobi Fields in a Symmetric Space, TOKYO J. MATH.VOL. 7, No. 1, 1984.

[56].Shlomo Sternberg, Lie algebras, Lecture notes, April 23, 2004.

[57].LIE GROUPS IN PHYSICS1 (version25/06/07) Institute for Theoretical Physics Utrecht University (English version by G. 't Hooft Original text by M.J.G. Veltman B.Q.P.J. de Wit and G. 't Hooft Beta Faculty 2007.

[58].E.Koorambas, (in preparation)

[59].M.Bugden, A Tour of T-duality Geometric and Topological Aspects of T-dualities, PhD thesis, Mathematical Sciences Institute Australian National University, Australian August 2018

[60].A. Douady and M. Lazard, Espaces fibr'es en alg 'ebres de Lie et en groupes, Invent. Math. 1 (1966), 133-151

[61].R. Foot, Generalized mirror matter models, Physics Letters B 632 (2006) 467-470.

[62] R. Foot, Int. J. Mod. Phys. A 19 (2004) 3807, astro-ph/0309330.

[63] R. Foot, H. Lew, R.R. Volkas, Mod. Phys. Lett. A 7 (1992) 2567; R. Foot, Mod. Phys. Lett. A 9 (1994) 169, hep-ph/9402241; R. Foot, R.R. Volkas, Phys. Rev. D 52 (1995) 6595, hep-ph/9505359.

[64].E.Koorambas, (in preparation)

[65]. Churchill, Ruel V. and Brown, James Ward Complex variables and applications McGraw-Hill Book Co. New York 1984.

[66]. Conway, John B. Functions of one complex variable Graduate Texts in Mathematics SpringerVerlag, New York 1978.

[67]. Jones, Gareth A. and Singerman, David Complex functions - An algebraic and geometric viewpoint Cambridge University Press, Cambridge 1987.

[68]. Saff, Edward B. and Snider, Arthur David Fundamentals of complex analysis with applications to engineering, Science, and Mathematics Prentice Hall. 2003

[69].X.Song Quantum Cellular Automata Models for General Dirac Equation, arXiv:1610.02425 [quant-ph] (2019) 
[70].Wen- Long Lin. Chinese Journal of Physics 6, (4), 1978.

[71].D. Meyer, From Quantum Cellular Automata to Quantum Lattice Gases, J. Stat. Phys. 85 (1996) 551574.

[72].M F C Martins Quintela, J M B Lopes dos Santos 2021 J. Phys.: Condens. Matter 33035901

[73].M. Quintela. From the 1D Schrödinger infinite well to Dirac-Weyl graphene flakes. Master's thesis, Faculdade de Ciências, Universidade do Porto, 2019.

[74].R.B. Bhat. Flexural vibration of polygonal plates using characteristic orthogonal polynomials in two variables. Journal of Sound and Vibration, 114(1):65-71, January 1987.

[75].V Amar, M Pauri, and A Scotti. Schrödinger-equation For Convex Plane Polygons .2. A Nogo Theorem For Plane-waves Representation Of Solutions. Journal Of Mathematical Physics, 34(8):33433350, Aug 1993.

[76].T.Koshy, Parity and Primality of Catalan Numbers Thomas Koshy VOL. 37, NO. 1, JANUARY 2006 THE COLLEGE MATHEMATICS JOURNAL 53

[78]. M. Gardner, Catalan Numbers: An Integer sequence that Materializes in Unexpected Places, Scientific Amer ican, 234 (1976) 120-125.

[79]. R. K. Guy, The Second Strong Law of Small Numbers, Mathematics Magazine, 63 (1990) 3-20.

[80]. T. Koshy, Discrete Mathematics with Applications, Elsevier Academic Press, 2004. 